\title{
Nonlinear rheology of colloidal gels with intermediate volume fraction
}

\author{
M. Laurati and S. U. Egelhaaf \\ Condensed Matter Physics Laboratory, Heinrich-Heine University, \\ Universitätsstraße 1, 40225 Düsseldorf, Germany \\ G. Petekidis ${ }^{\text {a) }}$ \\ Department of Materials Science and Technology and IESL-FORTH, \\ University of Crete, Heraklion, Greece
}

(Received 23 July 2010; final revision received 30 November 2010; published 4 April 2011)

\begin{abstract}
Synopsis
The depletion attraction, induced upon addition of a nonadsorbing polymer to a colloidal solution, can lead to gel formation at sufficiently high polymer concentrations, which corresponds to strong attractive interactions. We have investigated the nonlinear rheological response, in particular the yielding, of colloidal gels with an intermediate volume fraction and variable interparticle attraction. Two distinct yielding processes are observed in both oscillatory experiments, namely, dynamic strain sweeps and transient experiments, here step rate, creep, and recovery tests. The first yielding process occurs at strains similar to the range of the interparticle potential and is interpreted as the breaking of bonds, which destroys the particle network and leads to individual clusters. The process of bond breaking is successfully modeled as the escape of a particle from the potential well of its nearest neighbor. The second yield point occurs at larger strains and is related to the deformation and fragmentation of clusters, consistent with the observed dependence of the yield strain on attraction. Both yield stresses exhibit a power-law dependence on attraction strength in agreement with observations of other systems and theoretical predictions. Furthermore, the observed two-step yielding reveals similarities, and also differences, to the rheology of attractive colloidal glasses. (C) 2011 The Society of Rheology. [DOI: 10.1122/1.3571554]
\end{abstract}

\section{INTRODUCTION}

Viscoelastic materials are frequently subjected to large deformations as evidenced in many industrial processes and in our daily lives when eating food or using personal care products [Coussot (2005); Larson (1999)]. Upon an applied deformation or stress, many viscoelastic materials respond elastically, i.e., solidlike, below a certain strain or stress. Beyond this characteristic yield strain or stress the response is dissipative, i.e., fluidlike. This behavior is known as yielding and occurs in very different materials, ranging from complex fluids to amorphous metals [Becu et al. (2006); Brader et al. (2007, 2008); Carrier and Petekidis (2009); Crassous et al. (2008); Fuchs and Ballauff

\footnotetext{
${ }^{a)}$ Electronic mail: georgp@iesl.forth.gr
} 
(2005); Gopalakrishnan and Zukoski (2007); Johnson and Samwer (2005); Le Grand and Petekidis (2008); Miyazaki and Reichman (2002); Miyazaki et al. (2006); Pham et al. (2006, 2008); Rao et al. (2006); Rottler and Robbins (2003); Rouyer et al. (2003); Siebenburger et al. (2009); Stokes and Frith (2008); Walls et al. (2003)]. A thorough understanding of yielding, however, is still missing.

The yielding of dynamically arrested states, namely, colloidal glasses and gels, has recently attracted particular interest. In suspensions of colloidal hard spheres with volume fractions $\phi \geq 0.58$, an amorphous solid state, a repulsive glass, has been found [Pusey (1991); Pusey and van Megen $(1986,1987)]$. If large enough interparticle attractions are present, amorphous solid states can be formed at any volume fraction: Attractive glasses at high colloid volume fractions [Dawson et al. (2001); Eckert and Bartsch (2002); Pham et al. $(2002,2004)$; Poon et al. (2003)] are usually distinguished from gels at lower volume fractions [Poon (2002); Shah et al. (2003a)]. The location of the transition from a fluid to an attractive glass or gel can be predicted by mode-coupling theory (MCT) and is consistent with experiments [Bergenholtz et al. (2003); Laurati et al. (2009); Shah et al. (2003b)]. It has been suggested that dynamical arrest in these two systems is driven by different mechanisms: bonding in attractive glasses as described by MCT [Cates (2003); Dawson et al. (2001); Pham et al. (2002)] and phase separation in low volume fraction gels [Cardinaux et al. (2007); Ilett et al. (1995); Lu et al. (2008); Manley et al. (2005); Poon et al. (1997); Verduin and Dhont (1995); Verhaegh et al. (1997)]. However, the formation of gels has also been described as a glass transition of clusters by a MCT-based approach [Kroy et al. (2004)]. This suggests that the mechanical properties of gels are governed by the cluster length scale, and those of attractive glasses are governed by the particle size [Zaccone and Del Gado (2009)]. The distinction between attractive glasses and gels becomes, however, difficult to describe at intermediate volume fractions.

The rheology of repulsive and attractive glasses has been studied both experimentally [Conrad et al. (2010); Crassous et al. (2008); Fuchs and Ballauff (2005); Miyazaki et al. (2006); Petekidis et al. (2004); Pham et al. (2006, 2008); Shah et al. (2003b); Varadan and Solomon (2003)] and theoretically [Brader et al. (2007, 2008); Fuchs and Ballauff (2005); Fuchs and Cates (2002); Miyazaki and Reichman (2002); Miyazaki et al. (2006); Sollich (1998); Zaccone and Del Gado (2009)]. Repulsive glasses show a single yielding process [Petekidis et al. (2002a, 2003); Pham et al. (2008)]. This has been associated with the accelerated decay of structural correlations due to shear advection by MCT [Brader et al. (2007); Crassous et al. (2008); Fuchs and Cates (2002); Miyazaki and Reichman (2002); Miyazaki et al. (2006)] and with particle delocalization due to hopping processes by trap models [Dyre (1987); Monthus and Bouchaud (1996); Sollich (1998)]. These two approaches have also been combined [Kobelev and Schweizer (2005b)]: The nonergodicity transition of MCT is used to determine the onset of particle localization due to the emergence of finite free-energy barriers in the energy landscape. The importance of nonlinear contributions in achieving a comprehensive theoretical understanding of the stress-strain relation from nonlinear rheology of dense colloidal suspensions under time-dependent shear has recently been recognized [Brader et al. $(2007,2008)$; Fuchs and Cates (2002)], while experimental evidence exists both from steady and oscillatory measurements in a variety of systems [Carrier and Petekidis (2009); Crassous et al. (2008); Gadala-Maria and Acrivos (1980); Helgeson et al. (2007); Hyun et al. (2007); Le Grand and Petekidis (2008); Siebenburger et al. (2009)].

Attractive glasses present an even more complex yielding behavior with two distinct yielding processes [Pham et al. $(2006,2008)]$. These have been associated with the breaking of interparticle bonds at small strains and with the limit of elastic cage deformations at large strains. To date, there is no theoretical description of these processes. 
Some effort has also been devoted to the study of the nonlinear rheology of colloidal gels, which include gels formed due to van der Waals attraction [Buscall et al. (1987); Furst and Pantina (2007); Kobelev and Schweizer (2005a); Larson (1999); Russel and Grant (2000)] or depletion interactions [Kobelev and Schweizer (2005a); Koumakis and Petekidis (2008)] and also thermoreversible gels [Gopalakrishnan and Zukoski (2007); Woutersen and de Kruif (1991)]. For strongly flocculated gels, a single yielding process with a weak dependence on volume fraction and attraction strength is observed [Buscall et al. (1987); Larson (1999); Russel and Grant (2000)], while thermoreversible gels show a stronger dependence on volume fraction [Gopalakrishnan and Zukoski (2007)], which agrees with the combined MCT-barrier hopping approach [Kobelev and Schweizer (2005a)]. In contrast, two yielding processes, as in attractive glasses, are found in depletion gels with intermediate volume fraction with a strong interplay as volume fraction is decreasing. The presence of structural heterogeneities and clusterlike structures over a broad range of intermediate volume fractions, as observed by scattering and microscopy [Dibble et al. (2006); Laurati et al. (2009); Shah et al. (2003c); Smith (2004); Varadan and Solomon (2003)], suggests that an important role is played by structural heterogeneities in the yielding of these systems.

Here, we investigate the nonlinear rheological properties, in particular the yielding, of depletion gels with intermediate volume fractions as a function of attraction strength. The strength of the attraction is varied by changing the concentration of the depletant, a nonadsorbing polymer. Changes in the interparticle interactions induce changes in the structure and dynamics, in particular in the size of heterogeneities, which have been determined by scattering and microscopy [Dibble et al. (2006); Laurati et al. (2009); Shah et al. (2003c); Smith et al. (2007); Varadan and Solomon (2003)]. The dependence of the size of heterogeneities on polymer concentration, which shows a maximum around the gelation boundary [Dibble et al. (2006); Laurati et al. (2009)], suggests a nonequilibrium route leading to gelation at intermediate volume fractions [ $\mathrm{Lu}$ et al. (2008)]. We investigate the effect of these structural and dynamic changes on shear-induced rearrangements and yielding by rheology, namely, dynamic strain sweeps (DSSs), step rate tests, flow curves, and creep and recovery experiments. Both the yield strain and stress were measured under different conditions and the data compared to theoretical predictions [Kobelev and Schweizer (2005a); Smith et al. (2007)]. These results together with investigations of attractive glasses at large volume fractions [Pham et al. $(2006,2008)$ ] and gels with a constant attraction strength but with variable volume fractions [Koumakis and Petekidis $(2008,2011)]$ provide a comprehensive mapping of nonlinear rheology of attractive glasses and gels.

\section{MATERIALS AND METHODS}

\section{A. Samples}

The samples contain mixtures of polymethylmethacrylate (PMMA) hard-sphere colloids and linear polystyrene (PS) dispersed in cis-decalin at a temperature $T=23{ }^{\circ} \mathrm{C}$. The average hydrodynamic radius of the PMMA particles, $R=137 \mathrm{~nm}$, was determined in very dilute samples by dynamic light scattering. The absence of crystallization in quiescent and sheared samples indicates a colloid polydispersity of at least $12 \%$. The radius of gyration of PS (molecular weight $M_{w}=132900 \mathrm{~g} / \mathrm{mol}$, from Polymer Laboratories) in cis-decalin is $r_{g}=10.8 \mathrm{~nm}$ [following Berry (1966)] and the polydispersity $M_{w} / M_{n}$ $=1.01$ as specified by the supplier. This implies a polymer-colloid size ratio $\xi=r_{g} / R$ $=0.079 \pm 0.013$ in dilute solution. The effective polymer-colloid size ratio $\xi^{*}$ has been calculated at a colloid volume fraction $\phi=0.4$ and different polymer concentrations 
TABLE I. Investigated samples, which are referred to by their nominal polymer concentration. $\phi$ is the colloid volume fraction; $c_{p} / c_{p}^{*}$ and $c_{p}^{\text {free }} / c_{p}^{*}$ are the polymer concentrations in the total and free volume, respectively, normalized by the overlap concentration $c_{p}^{*}$; $\xi^{*}$ is the effective polymer-colloid size ratio; and $U_{0}$ is the depth of the attractive potential.

\begin{tabular}{lccccc}
\hline \hline$c_{p} / c_{p}^{*}$ (nominal) & $\phi$ & $c_{p} / c_{p}^{*}$ & $c_{p}^{\text {free } / c_{p}^{*}}$ & $\xi^{*}$ & $U_{0} / k_{B} T$ \\
\hline 0 & 0.40 & & & & \\
0.1 & 0.40 & 0.10 & 0.21 & $0.079 \pm 0.013$ & -5.0 \\
0.2 & 0.40 & 0.20 & 0.40 & $0.067 \pm 0.009$ & -10.2 \\
0.25 & 0.40 & 0.25 & 0.49 & $0.063 \pm 0.008$ & -12.7 \\
0.32 & 0.39 & 0.32 & 0.62 & $0.057 \pm 0.007$ & -16.3 \\
0.4 & 0.40 & 0.40 & 0.76 & $0.052 \pm 0.007$ & -20.5 \\
0.5 & 0.41 & 0.48 & 0.90 & $0.048 \pm 0.006$ & -24.6 \\
0.7 & 0.40 & 0.70 & 1.28 & $0.040 \pm 0.006$ & -36.0 \\
0.8 & 0.40 & 0.82 & 1.48 & $0.037 \pm 0.005$ & -42.1 \\
1 & 0.40 & 0.99 & 1.78 & $0.033 \pm 0.005$ & -51.1 \\
1.5 & 0.40 & 1.49 & 2.63 & $0.027 \pm 0.004$ & -77.5 \\
2 & 0.40 & 1.99 & 3.48 & $0.023 \pm 0.004$ & -104.2 \\
\hline \hline
\end{tabular}

$0.0<c_{p} / c_{p}^{*} \leq 2.0$, where $c_{p}^{*}$ is the overlap concentration, taking into account the concentration dependence of the polymer size and the mesh size using the generalized free volume theory (GFVT) [Aarts et al. (2002); Fleer and Tuinier (2007); Lekkerkerker et al. (1992)] (Table I). The calculations are based on polymers in a good solvent, although our conditions approach those of a $\theta$ solvent, and thus might slightly overestimate the values of $\xi^{*}$. Furthermore, the Asakura-Oosawa (AO) description of the depletion potential is not strictly valid around and beyond the polymer overlap concentration $c_{p}^{*}$ [Fuchs and Schweizer (2002)]. The values of the depth of the attraction potential $U_{0}$ for large polymer concentrations, reported in Table I, have to be considered therefore as indicative.

The samples were prepared by mixing appropriate amounts of colloid and polymer stock solutions. The colloid stock solution with volume fraction $\phi=0.6$ was obtained by redispersing spun-down sediments, whose volume fraction was estimated to be $\phi=0.67$ when taking polydispersity into account [Schaertl and Silescu (1994)]. Polymer stock solutions of different concentrations were prepared by adding dry polymer to cis-decalin with the concentration $c_{p}$ (mass/volume) calculated from the weighed masses of the two components. After mixing, samples were vigorously shaken using a vortex shaker and then homogenized for 3 days in a rotating-wheel mixer. The compositions of the samples are summarized in Table I, with the first column listing the nominal polymer concentrations, which are used to refer to the samples in the following discussion. Values of the volume fraction $\phi$ and the polymer concentration $c_{p}$ refer to the total volume and $c_{p}^{\text {free }}$ to the volume not occupied by colloids, as estimated by GFVT [Aarts et al. (2002); Fleer and Tuinier (2007); Lekkerkerker et al. (1992)]. Polymer concentrations are normalized by the polymer overlap concentration $c_{p}^{*}=3 M_{w} / 4 \pi N_{A} r_{g}^{3}$, where $N_{A}$ is Avogadro's number.

\section{B. Rheology}

Strain-controlled rheological measurements were performed on an ARES-HR straincontrolled rheometer with a force balance transducer 10FRTN1 mainly using a cone-plate geometry (cone angle of $0.044 \mathrm{rad}$ and cone diameter of $25 \mathrm{~mm}$ ). Homemade mechanically roughened cone-plate fixtures (typically with a cone angle of 0.044 and a diameter of $25 \mathrm{~mm}$ and roughness of few hundred microns), calibrated with viscoelastic standards, 


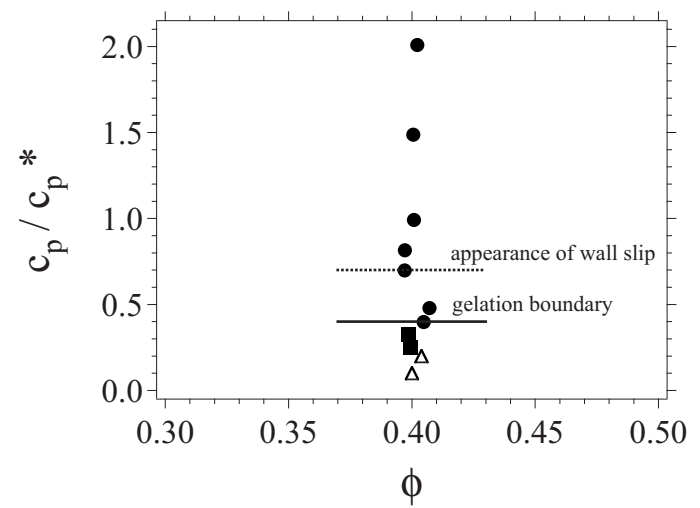

FIG. 1. Results from tube inversion of samples with similar volume fraction $\phi \approx 0.4$ but different polymer concentrations $c_{p} / c_{p}^{*}$. Samples exhibiting no flow (gels) $(\bullet)$, high viscosity fluids $(\boldsymbol{\square})$, and low viscosity fluids $(\triangle)$.

were also used to avoid wall slip (Sec. III B). Stress-controlled measurements were carried out using a DSR-200 rheometer (Rheometric Scientific, currently TA) with sandblasted cone-plate geometry (cone angle of $0.04 \mathrm{rad}$ and cone diameter of $50 \mathrm{~mm}$ ), which was coated with a mixture of small and large PMMA spheres (radii of about $150 \mathrm{~nm}$ and $1 \mu \mathrm{m}$, respectively) by sintering to obtain an irregular surface. (For the stress-controlled rheometer mechanically roughened geometries were not available.) This procedure has been shown to prevent wall slip in concentrated hard-sphere dispersions [Ballesta et al. (2008)]. Solvent evaporation was minimized by using a solvent saturation trap, which is designed to isolate the sample from the surrounding atmosphere by a fluid seal at the top and a permanent seal at the bottom. Inside the enclosure, solvent evaporation leads to a saturated atmosphere.

The effects of sample loading and aging were reduced by performing a standard rejuvenation procedure before each test: Directly after loading, we performed a dynamic strain or stress sweep (for strain or stress-controlled measurements, respectively), i.e., we applied oscillatory shear to the samples with a frequency $\omega=1 \mathrm{rad} / \mathrm{s}$ and an increasing strain amplitude $\gamma$ (or stress amplitude $\sigma$ ) until the sample showed a liquidlike response. In strain-controlled experiments, a strain amplitude $\gamma=8$ was large enough for all polymer concentrations, while in stress-controlled measurements stress amplitudes of $15 \leq \sigma \leq 60 \mathrm{~Pa}$ were used depending on the polymer concentration, $0.4 \leq c_{p} / c_{p}^{*} \leq 1.0$. Samples were then subjected to a dynamic time sweep with a frequency $\omega=1 \mathrm{rad} / \mathrm{s}$ and a strain $\gamma=8$ (or a stress $15 \leq \sigma \leq 60 \mathrm{~Pa}$ for stress-controlled measurements) until the elastic, $G^{\prime}$, and viscous, $G^{\prime \prime}$, moduli reached constant steady-state values. Subsequently, the samples were left at rest for a waiting time $t_{w}=300 \mathrm{~s}$ before the test was started. This waiting time was chosen to ensure a reproducible initial state, on the basis of the time dependence (aging) of the linear viscoelastic properties after rejuvenation.

\section{RESULTS AND DISCUSSION}

\section{A. Tube inversion}

The macroscopic behavior of the samples was studied by observing their flow behavior upon tube inversion. All samples had colloid volume fractions of $\phi \approx 0.4$, but different polymer concentrations $c_{p} / c_{p}^{*}$, where $c_{p}^{*}$ is the polymer overlap concentration (Table I and Fig. 1). Gel samples were identified initially by the absence of flow after tube inversion. 


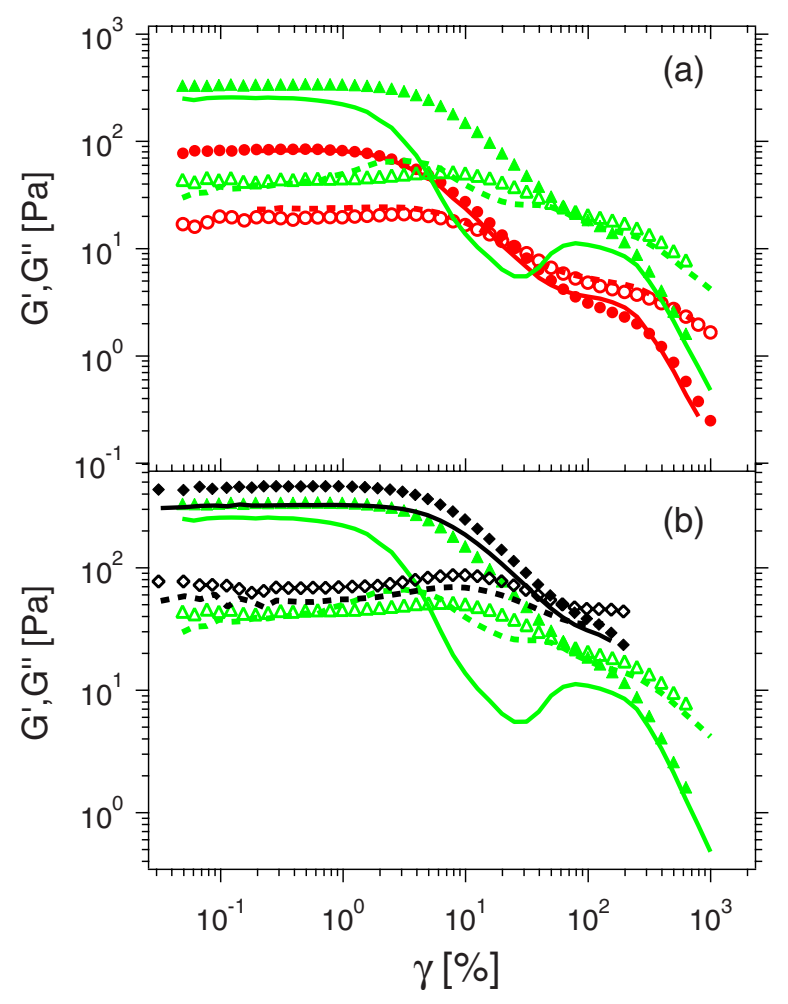

FIG. 2. Comparison of DSS measurements performed with roughened (symbols) and smooth (lines) cone-plate geometries. Full symbols and solid lines represent the elastic modulus $G^{\prime}$, while open symbols and dotted lines represent the viscous modulus $G^{\prime \prime}$. (a) Dependence on polymer concentration $\left[c_{p} / c_{p}^{*}=0.4(\mathbf{O})\right.$ and $\left.1.0(\mathbf{\Delta})\right]$ for a fixed frequency $\omega=1 \mathrm{rad} / \mathrm{s}$. (b) Dependence on frequency $[\omega=1 \mathrm{rad} / \mathrm{s}(\boldsymbol{\Delta})$ and $100 \mathrm{rad} / \mathrm{s}(\diamond)]$ for a fixed polymer concentration $c_{p} / c_{p}^{*}=1.0$.

This was observed for samples with $c_{p} / c_{p}^{*} \geq 0.4$. Samples with $0.2<c_{p} / c_{p}^{*}<0.4$ still flowed upon inversion but exhibited high viscosities, while samples with $c_{p} / c_{p}^{*} \leq 0.2$ showed low viscosities.

\section{B. Wall slip}

The response using smooth and mechanically roughened geometries to dynamic strain sweeps (DSS) is shown in Fig. 2. A comparison of the results for samples with different polymer concentrations $c_{p} / c_{p}^{*}$ at a fixed frequency $\omega=1 \mathrm{rad} / \mathrm{s}$ Fig. 2(a) shows that for $c_{p} / c_{p}^{*}=0.4$, comparable results are obtained with different degrees of surface roughness, while markedly different results are obtained for $c_{p} / c_{p}^{*}=1.0$ in the nonlinear regime. Indeed, for $c_{p} / c_{p}^{*}=1.0$ the storage modulus $G^{\prime}$ is smaller at all strains $\gamma$ when smooth surfaces are used. In addition, the strain dependences of both the elastic, $G^{\prime}$, and viscous, $G^{\prime \prime}$, moduli are qualitatively different. One particular difference when using smooth surfaces is in $G^{\prime}$; a minimum is evident at $\gamma \approx 30 \%$ followed by a maximum at $\gamma \approx 100 \%$. These features disappear when roughened geometries are used. For $c_{p} / c_{p}^{*}=1.0$, DSS measurements performed at different frequencies $\omega$ [Fig. 2(b)] indicate quantitative differences for different surface roughness with the differences in the qualitative trends becoming more pronounced at lower frequencies. Such discrepancies between the response using smooth and roughened geometries have been attributed to slip at the smooth 
surfaces [Walls et al. (2003)], while the frequency dependence is in qualitative agreement with findings in colloidal glasses under steady shear where slip is observed at low rates [Ballesta et al. (2008)]. Responses similar to those shown for $c_{p} / c_{p}^{*}=1.0$ were measured for samples with $c_{p} / c_{p}^{*} \geq 0.7$. Slip during oscillatory measurements was also detectable either by the existence of even harmonics in the stress signal (typically around 2\%) or by microscopic dynamics measured with the light-scattering echo (LS-echo) technique [Petekidis et al. (2002a, 2002b)], as will be reported in detail elsewhere.

Wall slip thus strongly influences the measurements at large strains in the nonlinear regime for samples with polymer concentrations $c_{p} / c_{p}^{*} \geq 0.7$ (as indicated in Fig. 1), but has little effect on the linear rheological response at small strains. All measurements reported here (and previously performed to investigate the linear response [Laurati et al. (2009)]) have been done with roughened surfaces to minimize wall slip. As our experiments indicate, the results with roughened and smooth surfaces agree where wall slip can be excluded.

Besides wall slip, shear banding has been observed in colloidal glasses under steady shear [Besseling et al. (2010)] and colloidal crystals under oscillatory shear [Cohen et al. (2006)]. A direct detection of such phenomena requires monitoring the velocity profile during shear [Ravindranath and Wang (2008); Tapadia et al. (2006)]. Preliminary confocal microscopy experiments under steady shear on gels similar to the ones investigated here have revealed shear banding only at quite low shear rates and high attraction strengths [Ballesta et al. (in preparation)]. However, no oscillatory shear experiments are presently available. An indication for the absence of nonperiodic shear banding effects during large amplitude oscillatory shear (LAOS) was obtained by Fourier transform (FT) analysis of the stress response, where even harmonics were absent [Laurati et al. (in preparation)]. Hence, although shear banding cannot be entirely excluded, indirect evidence suggests that it is absent in the current LAOS measurements.

\section{Linear rheological response}

Linear viscoelastic measurements presented before in the same system [Laurati et al. (2009)] are briefly summarized here to serve as a reference for the discussion of the nonlinear viscoelastic properties. Dynamic frequency sweep (DFS) tests have been performed with samples below and above the macroscopic gelation boundary.

Below the macroscopic gelation boundary [Fig. 3(a)], the crossing points between the elastic (storage), $G^{\prime}$, and viscous (loss), $G^{\prime \prime}$, moduli shift toward smaller frequencies upon increasing the polymer concentration $c_{p} / c_{p}^{*}$. This indicates a transition from a liquidlike to a solidlike response which occurs at increasingly smaller frequencies $\omega$ (corresponding to larger characteristic times) as the polymer concentration, i.e., interparticle attraction, is increased. The characteristic time is related to the "bond" lifetime estimated by the time needed to escape from the attractive potential well. Bond breaking was found to be the dominant process close to but still below the gelation boundary, where the samples already have a network structure with a finite lifetime (transient network), while particle or cluster diffusion seems to control structural relaxation at the lowest polymer concentrations [Laurati et al. (2009)]. In this regime, i.e., below the gelation boundary, MCT successfully describes the dependence of the elastic modulus $G^{\prime}$ on polymer concentration [Fig. 3(c), blue line], while there are discrepancies in the frequency dependence [Laurati et al. (2009)].

In the gel region [Fig. 3(b)], solidlike behavior is observed, with $G^{\prime}$ being larger than $G^{\prime \prime}$ for all investigated frequencies and weakly frequency-dependent. The frequency dependence of $G^{\prime \prime}$ displays a minimum, indicating structural relaxation of the gels at long 

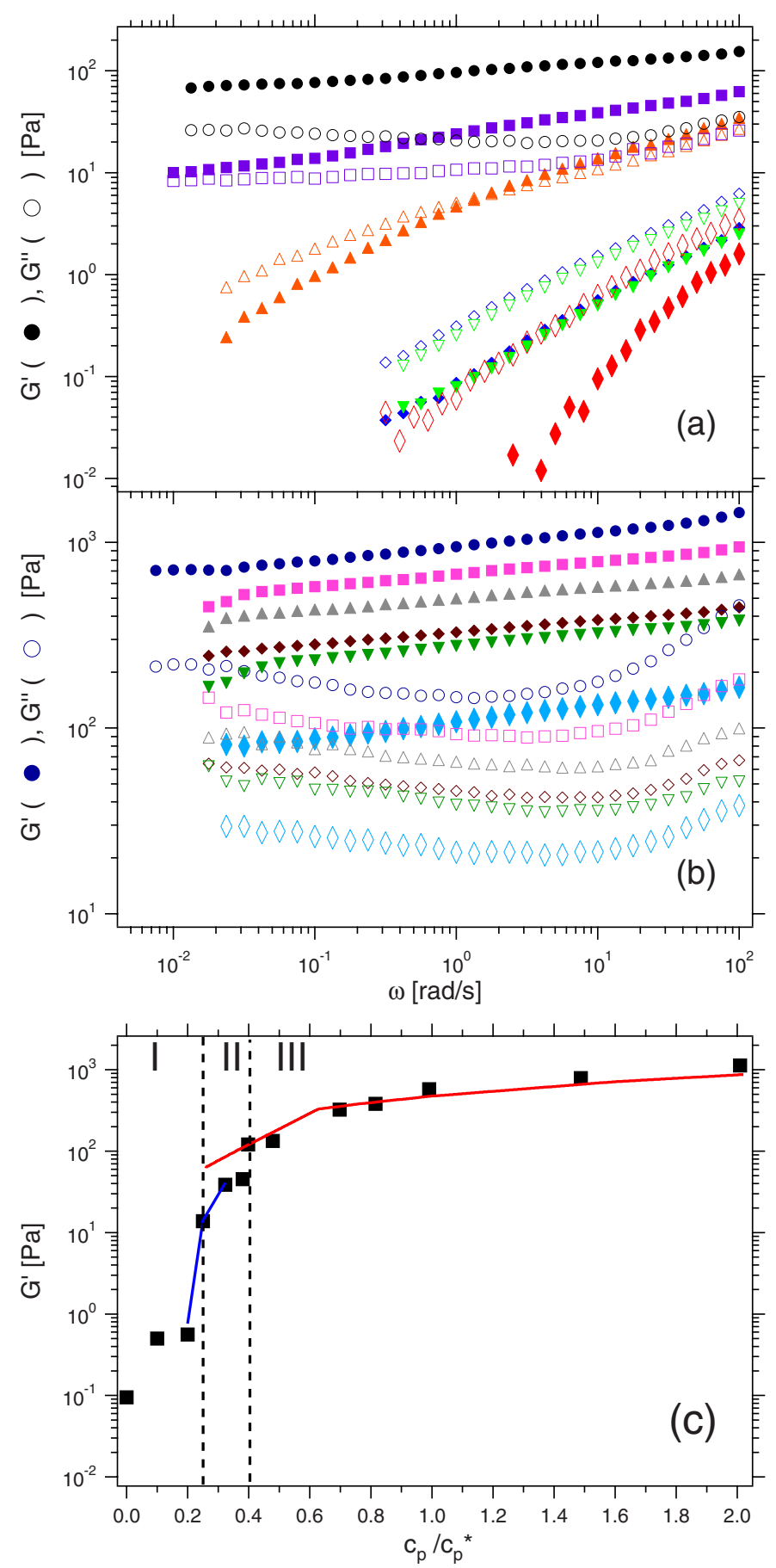

FIG. 3. DFSs with elastic (storage) modulus $G^{\prime}$ (filled symbols) and viscous (loss) modulus $G^{\prime \prime}$ (open symbols) as a function of frequency $\omega$. Samples (a) below and (b) above the macroscopic gelation boundary $\left(c_{p} / c_{p}^{*}\right.$ $=0.4)$ are shown with polymer concentrations $c_{p} / c_{p}^{*}=0(\diamond), 0.1(\boldsymbol{\nabla}), 0.2(\bullet), 0.25(\boldsymbol{\Delta}), 0.32(\boldsymbol{\square}), 0.4(\boldsymbol{\bullet}), 0.5$ $(\diamond), 0.7(\boldsymbol{\nabla}), 0.8(\diamond), 1.0(\mathbf{\Delta}), 1.5(\mathbf{\square})$, and $2.0(\boldsymbol{\bullet})$. (c) Elastic modulus $G^{\prime}$ extracted from DFS as a function of polymer concentration $c_{p} / c_{p}^{*}$ at frequency $\omega=10 \mathrm{rad} / \mathrm{s}(\boldsymbol{\square})$. Dashed lines separate the different states: fluid (I), transient network (II), and gel (III). Solid lines represent theoretical predictions for the elastic modulus of transient networks (blue line, Laurati et al., 2009) and gels (red line, Zaccone and Del Gado, 2009). 


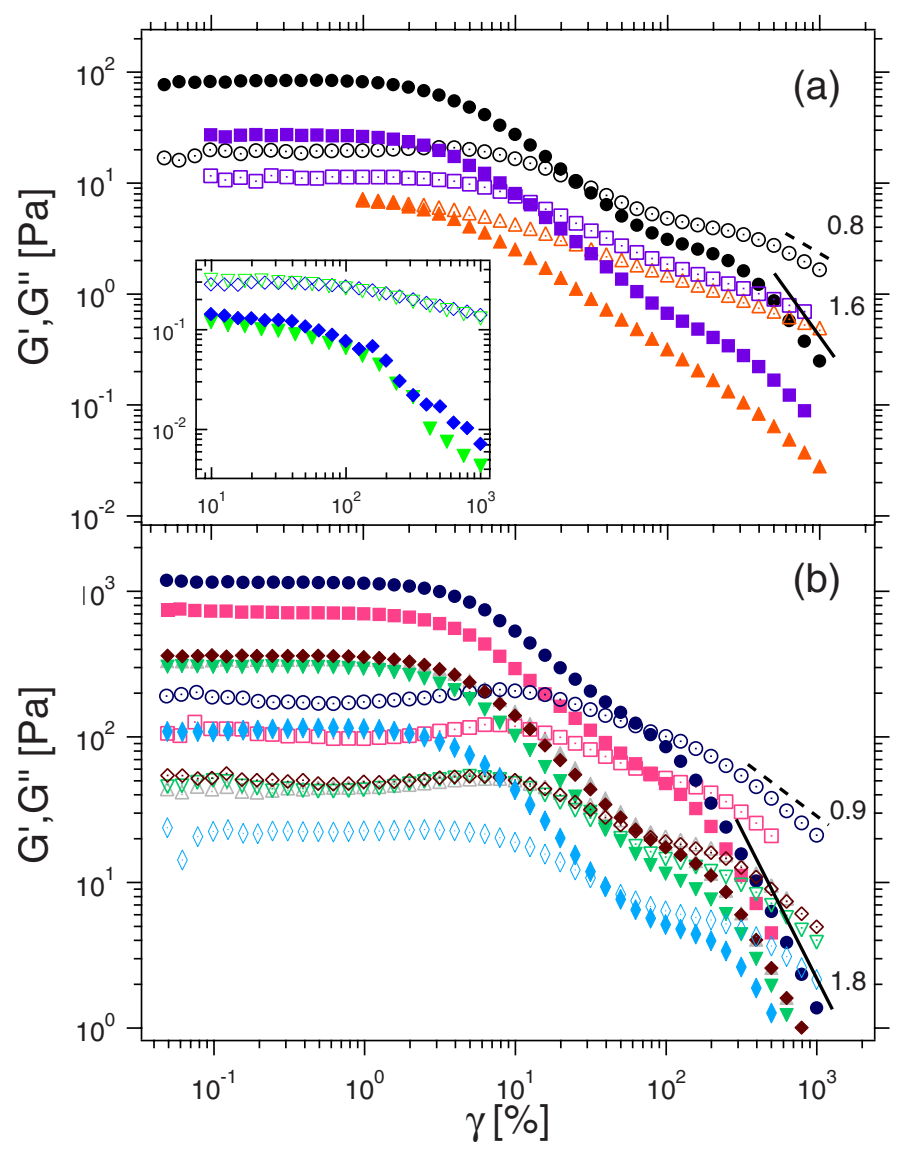

FIG. 4. DSSs with elastic (storage) modulus $G^{\prime}$ (filled symbols) and viscous (loss) modulus $G^{\prime \prime}$ (open symbols) as a function of strain $\gamma$ at $\omega=1 \mathrm{rad} / \mathrm{s}$. Samples (a) below and (b) above the macroscopic gelation boundary $\left(c_{p} / c_{p}^{*}=0.4\right)$ are shown with polymer concentrations $c_{p} / c_{p}^{*}=0.1(\boldsymbol{\nabla}$, inset), $0.2(\bullet$, inset), $0.25(\boldsymbol{\Delta}), 0.32(\boldsymbol{\nabla})$, $0.4(\bullet), 0.5(\diamond), 0.7(\nabla), 0.8(\bullet), 1.0(\mathbf{\Delta}), 1.5(\mathbf{\square})$, and $2.0(\bullet)$.

times. The almost linear dependence of $G^{\prime}$ on polymer concentration in this region [Fig. $3(\mathrm{c})$, region III] was attributed to the dominant contribution of intercluster links; this was theoretically confirmed recently [Zaccone and Del Gado (2009)] [Fig. 3(c), red line]. The intermediate volume fraction gel was modeled as a dispersion of clusters. The intracluster bonds were assumed to be permanent, and the elastic modulus of the gel thus determined by the intercluster elastic constant was calculated on the basis of the interaction potential as well as the coordination number, effective volume fraction, and characteristic size of the clusters [Zaccone and Del Gado (2009)].

\section{Nonlinear rheological response}

\section{DSSs}

DSSs at a frequency $\omega=1 \mathrm{rad} / \mathrm{s}$ were performed with samples below and above the macroscopic gelation boundary [Figs. 4(a) and 4(b), respectively]. The moduli measured in DSS tests represent the average response of the samples. The detailed nonlinear response can be accessed by analysis of the higher order harmonics and allows for a more detailed understanding of the intracycle yielding mechanisms [Ewoldt et al. (2008); 


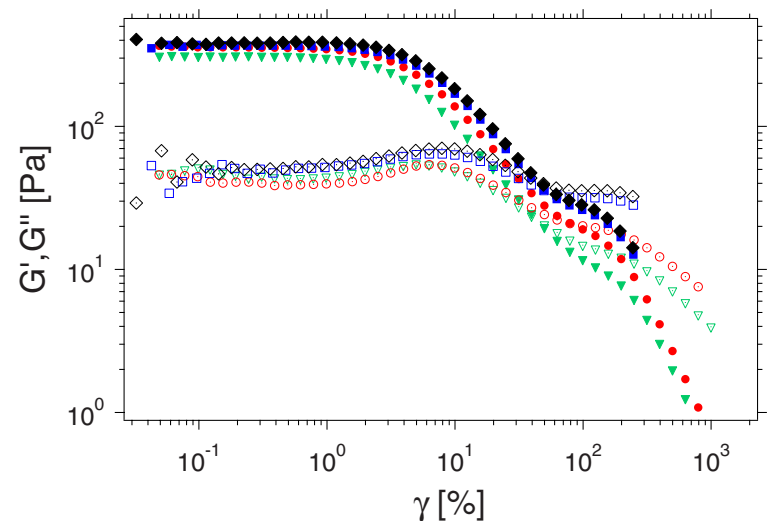

FIG. 5. DSSs with elastic (storage) modulus $G^{\prime}$ (filled symbols) and viscous (loss) modulus $G^{\prime \prime}$ (open symbols) as a function of strain $\gamma$ for a polymer concentration $c_{p} / c_{p}^{*}=0.7$ and frequencies $\omega=1 \mathrm{rad} / \mathrm{s}(\boldsymbol{\nabla}), 10 \mathrm{rad} / \mathrm{s}(\boldsymbol{\bullet})$, $69 \mathrm{rad} / \mathrm{s}(\mathbf{\square})$, and $100 \mathrm{rad} / \mathrm{s}(\bullet)$.

Renou et al. (2010)]. Such an analysis, performed on a selection of the samples and well inside the nonlinear regime, revealed amplitudes of the third harmonic as high as $20 \%$ and will be the subject of a forthcoming publication. Samples with polymer concentrations $c_{p} / c_{p}^{*}=0.1$ and 0.2 show a fluidlike response in agreement with the linear viscoelastic data [Fig. 3(a)], with shear thinning behavior at high strains. For $c_{p} / c_{p}^{*}=0.25, G^{\prime}$ is smaller than $G^{\prime \prime}$ at large strains, but becomes very similar below $\gamma \approx 2 \%$, i.e., in the linear regime. The response is thus intermediate between a highly viscous fluid and a low yield-stress solid and may also be used to determine the liquid-gel transition. This is attributed to a transient network structure, which is also consistent with its linear response [Laurati et al. (2009)]. At frequencies $\omega>1 \mathrm{rad} / \mathrm{s}$ (not shown), $G^{\prime}$ becomes larger than $G^{\prime \prime}$ in the linear regime, in agreement with DFS [Fig. 3(a), $c_{p} / c_{p}^{*}=0.25$ ].

For larger polymer concentrations $\left(c_{p} / c_{p}^{*} \geq 0.32\right), G^{\prime}$ is larger than $G^{\prime \prime}$ in the linear regime, and thus samples behave solidlike at low frequencies. Entering the nonlinear regime, the shear moduli decrease with increasing strain, with $G^{\prime}$ showing a stronger decrease than $G^{\prime \prime}$, which leads to a crossover between $G^{\prime}$ and $G^{\prime \prime}$ at strains $\gamma \gtrsim 10 \%$. Such a crossover in LAOS is often interpreted as a yielding point since at strains beyond the crossover many materials, for example, hard-sphere glasses [Derec et al. (2003); Mason and Weitz (1995); Miyazaki et al. (2006); Pham et al. (2006, 2008)] and other soft glassy materials [Carrier and Petekidis (2009); Cloitre et al. (2000); Daniel et al. (2001); Helgeson et al. (2007); Hyun et al. (2002); Le Grand and Petekidis (2008); Sollich (1998); Wyss et al. (2007)], show a fluidlike response with $G^{\prime} \sim \gamma^{\nu_{0}}$ and $G^{\prime \prime} \sim \gamma^{\nu_{1}}$ where $\nu_{0}$ $\approx 2 \nu_{1}$. In the present case, however, such a fluidlike response is not observed immediately after the crossover. Instead, over a broad range of strains the decay of the two moduli is weaker, indicating that some residual structure remains under flow. Only for $\gamma>100 \%$ is a strain regime with $\nu_{0} \approx 2 \nu_{1}$ observed with $-1.8 \leq \nu_{0} \leq-1.4$. Such a response indicates the existence of two different yielding mechanisms.

DSSs show a similar frequency dependence for all polymer concentrations investigated; Fig. 5 shows data for $c_{p} / c_{p}^{*}=0.7$. Both $G^{\prime}$ and $G^{\prime \prime}$ increase with increasing frequency $\omega$ in the linear and nonlinear regimes (except the 1 and $10 \mathrm{rad} / \mathrm{s}$ values of $G^{\prime \prime}$ in the linear regime, which show the opposite trends but are very similar). The strain dependence of $G^{\prime}$ and $G^{\prime \prime}$ does not change significantly with frequency, except for strains 


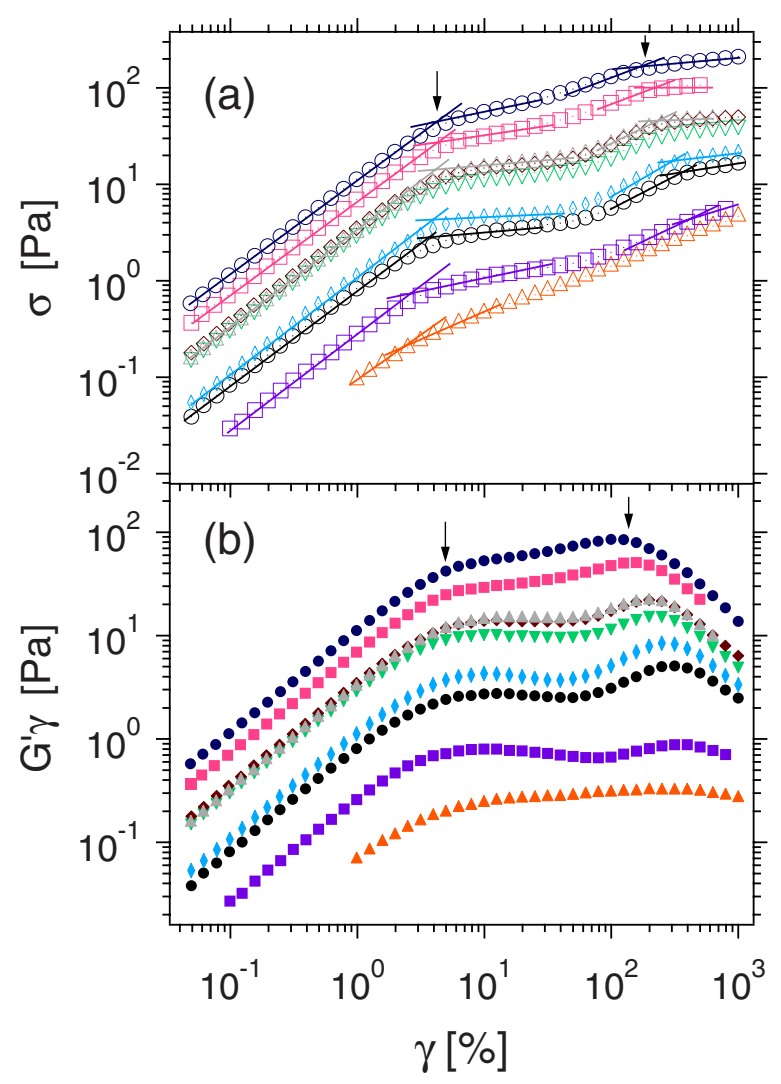

FIG. 6. DSSs represented as (a) total stress $\sigma$ and (b) its elastic component $G^{\prime} \gamma$ as a function of strain $\gamma$, which were performed at a frequency $\omega=1 \mathrm{rad} / \mathrm{s}$ for different polymer concentrations $c_{p} / c_{p}^{*}$ $=0.25,0.32,0.4,0.5,0.7,0.8,1.0,1.5,2.0$ (from bottom to top, same data and symbols as in Fig. 4). Yield points (indicated by arrows) are identified from (a) the inflection points in the stress response, located through the crossing points of the corresponding tangents, and (b) the maxima or shoulders in the elastic stress-strain curve.

$\gamma>100 \%$ where, with increasing frequency $\omega$, both moduli show a weaker decay prior to the final shear thinning regime (Fig. 5). The two yielding processes thus remain for all frequencies.

An alternative representation of the DSS data is to plot the strain dependence of the total stress $\sigma$ and its elastic component $G^{\prime} \gamma$ (Fig. 6). In this representation the two yielding mechanisms inferred from the trends of the shear moduli in Fig. 4 can be quantitatively determined as the inflection points in the strain dependence of the total stress $\sigma(\gamma)$, at which the stress-strain relation becomes increasingly sublinear [Fig. 6(a)]. The maxima or shoulders of the elastic component of the stress $G^{\prime} \gamma$ [Fig. 6(b), arrows] provide an alternative way of locating the yield points, as has been done for other soft matter systems [Kobelev and Schweizer (2005a); Pai and Khan (2002); Yang et al. (1986)]. Both $\sigma(\gamma)$ and $G^{\prime} \gamma(\gamma)$ support the presence of two yielding processes for samples with polymer concentrations $c_{p} / c_{p}^{*} \geq 0.32$.

Two yielding processes have also been observed for attractive glasses [Pham et al. (2006, 2008)]. In this case, the yielding mechanisms have been identified from distinct maxima of $G^{\prime \prime}$, i.e., maxima of energy dissipation, and are associated with bond breaking (at low strains of about 5\%) and the limit of elastic cage distortions (at large strains of about $50 \%$ ). For the gels studied here, the first yield point can also be associated with 


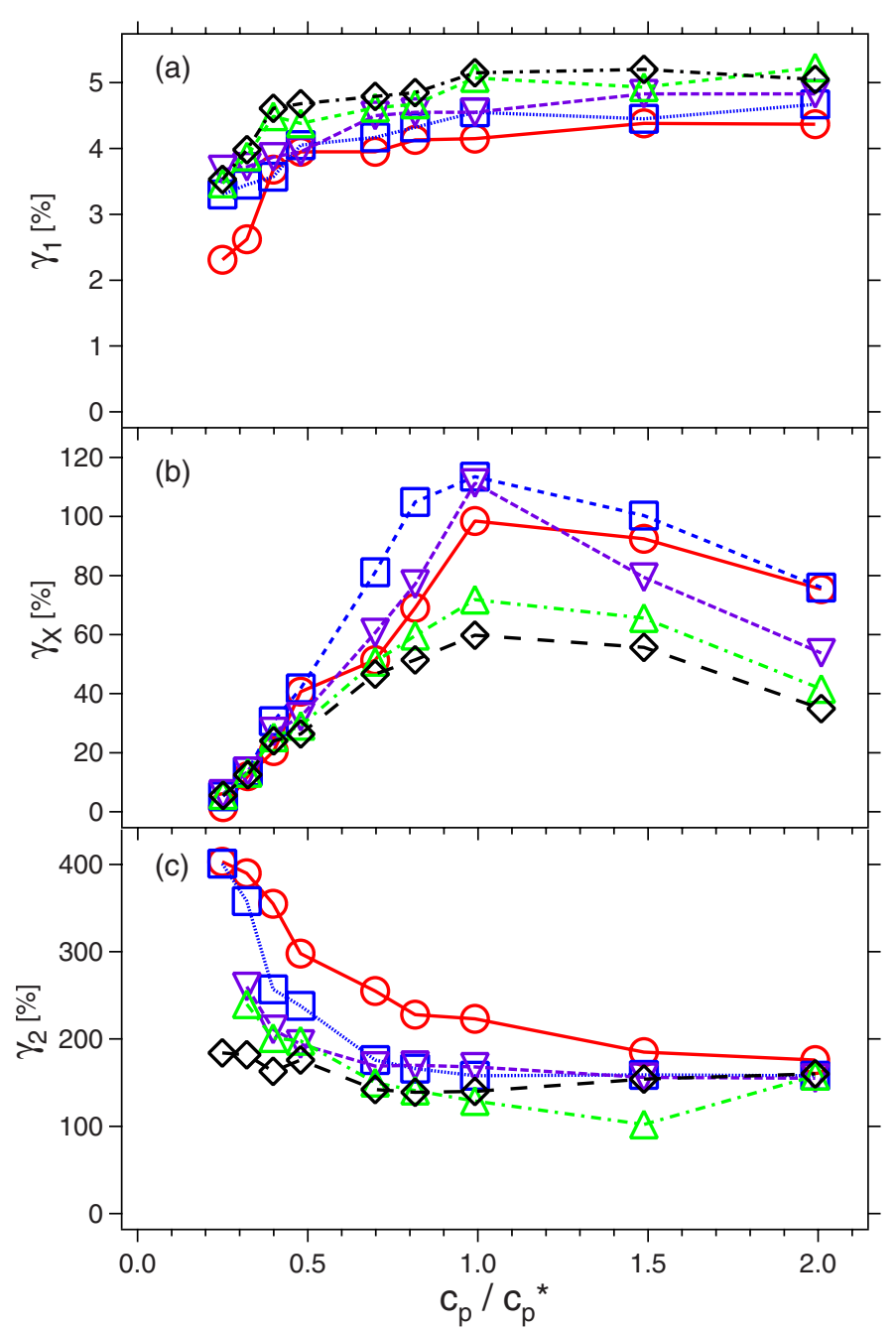

FIG. 7. Yield strains at (a) the first yield point, $\gamma_{1}$; (b) the crossing point, $\gamma_{X}$; and (c) the second yield point, $\gamma_{2}$, as a function of polymer concentration $c_{p} / c_{p}^{*}$ for different frequencies $\omega=1 \mathrm{rad} / \mathrm{s}(\bigcirc), 10 \mathrm{rad} / \mathrm{s}(\square), 40 \mathrm{rad} / \mathrm{s}$ $(\nabla), 69 \mathrm{rad} / \mathrm{s}(\triangle)$, and $100 \mathrm{rad} / \mathrm{s}(\diamond)$. The yield strains have been extracted from the DSSs with (a) the first yield point estimated as the onset of sublinear stress-strain relation, (b) the crossing point extracted from the strain dependence of $G^{\prime}$ and $G^{\prime \prime}$, and (c) the second yield point identified with the second inflection point of the stress-strain curve.

bond breaking, where the absence of a maximum at low polymer concentrations is consistent with the low strength of the bonds. The second weaker yield point, in contrast to glasses, relates to a more gradual restructuring resulting in a fluidized state. In the following, we compare this suggestion with our experimental data to test for consistency and to extract further information.

We now consider the dependence of the two yield points, as well as the crossing point between $G^{\prime}$ and $G^{\prime \prime}$, on the polymer concentration $c_{p} / c_{p}^{*}$ (or depletion attraction) and frequency $\omega$ and discuss the corresponding yield strains and yield stresses (Figs. 7 and 8). The first yield point reported $\left(\gamma_{1}, \sigma_{1}\right)$ corresponds to the onset of the sublinear stressstrain relation in the stress-strain plot [Fig. 6(a)]. We chose these values as they are better determined than the values obtained from the maximum or the onset of monotonous 


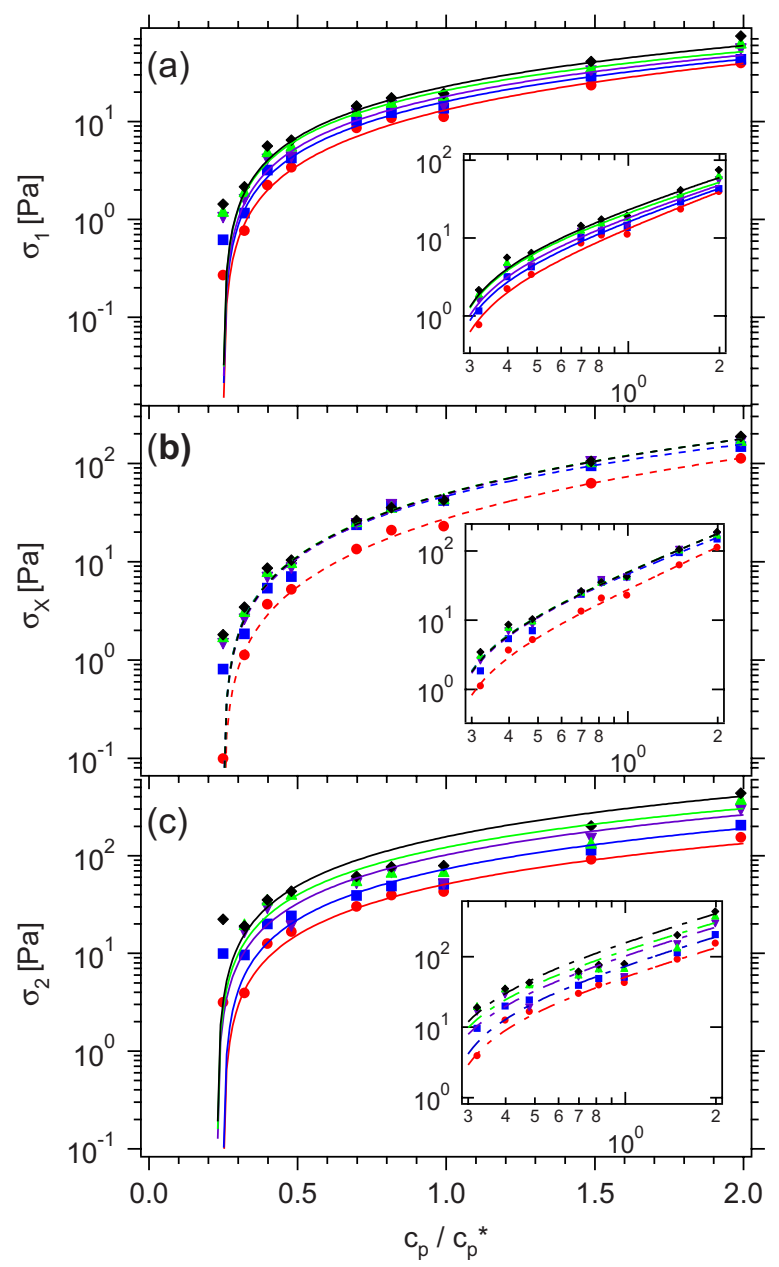

FIG. 8. Yield stresses at (a) the first yield point, $\sigma_{1}$; (b) the crossing point, $\sigma_{X}$; and (c) the second yield point, $\sigma_{2}$, as a function of polymer concentration $c_{p} / c_{p}^{*}$ for different frequencies $\omega=1 \mathrm{rad} / \mathrm{s}(\mathbf{\bullet}), 10 \mathrm{rad} / \mathrm{s}(\boldsymbol{\square}), 40$ $\mathrm{rad} / \mathrm{s}(\boldsymbol{\nabla}), 69 \mathrm{rad} / \mathrm{s}(\boldsymbol{\Delta})$, and $100 \mathrm{rad} / \mathrm{s}(\bullet)$. The yield stresses have been extracted from the DSSs with (a) the first yield point estimated as the onset of sublinear stress-strain relation, (b) the crossing point extracted from the strain dependence of $G^{\prime}$ and $G^{\prime \prime}$, and (c) the second yield point identified with the second inflection point of the stress-strain curve. Lines are fits to a power-law dependence $\sigma=\hat{\sigma}\left[\left(c_{p} / c_{p}^{\mathrm{gel}}\right)^{\mu}-1\right]$ (Kobelev and Schweizer, 2005a). Insets: Log-log plots of the same data.

decrease of $G^{\prime \prime}$ (Fig. 4), and the maximum or shoulder of the elastic stress $G^{\prime} \gamma$ [Fig. 6(b)], which show a comparable dependence on $c_{p} / c_{p}^{*}$ with only small differences in the absolute values. In comparison, the second yield point $\left(\gamma_{2}, \sigma_{2}\right)$ is identified with the second inflection of the stress-strain curve [Fig. 6(a)]. The crossing point $\left(\gamma_{X}, \sigma_{X}\right)$ is extracted from the strain dependence of $G^{\prime}$ and $G^{\prime \prime}$, at $G^{\prime}=G^{\prime \prime}$ (Fig. 4).

The first yield strain $\gamma_{1}$ increases in value with increasing polymer concentration [Fig. 7(a)]. At a low polymer concentration of $c_{p} / c_{p}^{*}=0.25$, it ranges, depending on frequency $\omega$, from $2.3 \% \lessgtr \gamma_{1} \leqslant 3.7 \%$ and then increases to $4.2 \% \lessgtr \gamma_{1} \lesssim 5.2 \%$, with the saturation value reached around $c_{p} / c_{p}^{*} \approx 1$. With increasing frequency $\omega$, the dependence of $\gamma_{1}$ on polymer concentration is qualitatively unchanged, but the value of $\gamma_{1}$ increases [Fig. 7(a)]. 
The values of the first yield strain $\gamma_{1}$ are comparable to the range of the attractive interparticle interactions; $\gamma_{1} \approx \xi^{*}$ (Table I). This suggests, by comparison to the behavior of attractive glasses [Pham et al. $(2006,2008)]$, that the first yield strain $\gamma_{1}$ reflects the breaking of interparticle bonds. This dissipates energy as indicated by the initial increase of $G^{\prime \prime}$ and leads to breaking of the space-spanning gel network into disconnected clusters. An underlying mechanism of such a process could be the breakage of bonds between particles that connect two clusters. Confocal microscopy studies of similar samples [Smith et al. (2007); Smith (2004)] suggest that a significant number of such particles are present. In such a picture, after intercluster bonds are broken, the system would represent a "cluster fluid." Cluster release would be responsible for the observed decrease of $G^{\prime}$ and $G^{\prime \prime}$ (Fig. 4) as well as the sublinear stress-strain relation [Fig. 6(a)]. Furthermore, the residual elasticity of the remaining clusters and the possibility of dynamically reforming bonds could cause a viscoelastic liquidlike response distinct from a simple Newtonian liquid. The presence of clusters or structural heterogeneities is consistent with scattering and microscopy results [Laurati et al. (2009)].

Although the values of the first yield strain $\gamma_{1}$ and the interaction range $\xi^{*}$ are comparable, their dependencies on polymer concentration display competing behavior; the interaction range decreases with increasing polymer concentration due to the transition from a dilute to a semidilute polymer solution. That the first yield strain $\gamma_{1}$ nevertheless increases with polymer concentration is attributed to the additional effect of the increasing interaction strength with increasing polymer concentration, which tends to suppress bond breaking. Below, we combine both effects, the range and strength of the attractive interactions, in a simple model that considers the escape from the attractive potential well of the nearest neighbors and leads to semiquantitative agreement with our data (Sec. IV).

The frequency dependence of the first yield strain $\gamma_{1}$ is attributed to the changing balance between shear-induced motion and Brownian motion. This is quantified by the Peclet number Pe, which describes the balance between Brownian motion, characterized in our case by the time required by the particle to diffuse over a distance corresponding to the interaction range of the potential $\left(\tau_{\mathrm{B}}=3.5 \times 10^{-4} \mathrm{~s}\right)$, and shear-induced motion, represented by the period of the oscillatory shear $(\omega \gamma)^{-1}$, with the strain amplitude $\gamma$. In our experiments, the Peclet number $\mathrm{Pe}=\omega \gamma \tau_{\mathrm{B}}$ is always smaller than 1, and thus Brownian motion dominates. This suggests that a particle bond, when it is considerably stretched by shear, has a high probability to break because Brownian motion allows the particle to escape from the attractive potential well. Within one period, the residence time of a particle in the stretched bond condition is determined by the frequency $\omega$, which implies that a higher frequency reduces the residence time and thus the probability of escape. Thus, a larger strain is required for higher frequencies. This qualitatively agrees with the observed increase in $\gamma_{1}$ with increasing frequency and is in semiquantitative agreement with the model proposed in Sec. IV.

In contrast to the first yield strain $\gamma_{1}$, the second yield strain $\gamma_{2}$ decreases with increasing polymer concentration [Fig. 7(c)]. At low polymer concentrations, $\gamma_{2}$ depends strongly on frequency $\omega$ with values ranging from about $200 \%$ to $400 \%$, while at higher polymer concentrations $\left(c_{p} / c_{p}^{*} \gtrsim 1\right)$ a value of $\gamma_{2} \approx 170 \%$ is attained, almost independent of frequency $\omega$. These strains correspond to displacements of about two to four particle diameters, depending on polymer concentration $c_{p} / c_{p}^{*}$. This length scale and its $c_{p} / c_{p}^{*}$-dependence are consistent with the typical cluster sizes observed by differential interference contrast (DIC) and confocal microscopy [Laurati et al. (2009); Smith (2004)]. In the same study, the shape of clusters composing the gel network was found to become more elongated with increasing polymer concentration. Similar trends for the dependence of the number of bonds and cluster shape on $c_{p} / c_{p}^{*}$ were observed by con- 
focal microscopy for PMMA-PS gels at a lower volume fraction, namely, $\phi=0.2$ [Dibble et al. (2006); Lee and Furst (2008)]. A response resembling that of a Newtonian liquid is thus only expected when not only the intercluster bonds are broken but also the clusters themselves are broken down or restructured. In order to quantitatively model this process, detailed structural information is required, which is not available at the moment. Qualitatively, cluster rearrangements occur on much larger length scales (about 150\%-400\%) than typical cage distortions of about $10 \%$ and $50 \%$ as implicated in the yielding of repulsive and attractive glasses, respectively [Pham et al. $(2006,2008)]$. This is consistent with the heterogeneous (cluster) structure of gels, whose length scale decreases with increasing volume fraction, i.e., by moving from gels to attractive glasses [Zaccone and Del Gado (2009)]. This connection is more precisely demonstrated in a recent investigation of the yielding of similar depletion gels along a line of constant attraction and the range of volume fractions covering attractive glasses as well as low volume fraction gels [Koumakis and Petekidis (2011)]. As in our experiments, this probes the effect of a decrease of the characteristic size of heterogeneities, which occurs with increasing attraction strength or polymer concentration [Dibble et al. (2006); Laurati et al. (2009); Lee and Furst (2008); Smith (2004)].

With increasing frequency $\omega$, the second yield strain $\gamma_{2}$ decreases, which indicates a decreasing size of the "free" clusters which are left after the first network disruption. This indicates an enhanced efficiency of shear in disintegrating the gel structure at larger frequencies or, in other words, a shear-rate dependence of such a cluster size.

The yield strain $\gamma_{X}$, which corresponds to the crossing point of $G^{\prime}$ and $G^{\prime \prime}$, first increases and then decreases as a function of polymer concentration, with a maximum around $c_{p} / c_{p}^{*} \approx 1$ [Fig. 7(b)]. The yield strain $\gamma_{X}$ is intermediate between the first, $\gamma_{1}$, and second, $\gamma_{2}$, yield strains, and thus the behavior is also expected to be intermediate, with the intercluster bonds broken and thus the gel network destroyed. However, not all intracluster bonds would be broken, and hence the clusters would not yet be completely restructured and a viscoelastic liquid of clusters would be present. The observed trend indicates a gradual transition from bond breaking to cluster rearrangements (around the gelation boundary, $\left.c_{p} / c_{p}^{*}=0.4\right)$ with the size of clusters decreasing upon increasing polymer concentration (within the gel region). The two processes take place at characteristic strains that become increasingly close with increasing $c_{p} / c_{p}^{*}$. Therefore, in close proximity to the gelation boundary, $\gamma_{X}$ follows the trend of the bond breaking process, while well inside the gel $\left(c_{p} / c_{p}^{*}>1\right)$ the processes begin to superimpose and $\gamma_{X}$ follows the smooth decrease of $\gamma_{2}$, with $\gamma_{1}$ being saturated. Similar trends have been predicted for the yield strain by a model for the nonlinear viscoelasticity of depletion gels [Kobelev and Schweizer (2005a)]. Note also that the trend of $\gamma_{X}$ reflects the ratio between $G^{\prime}$ and $G^{\prime \prime}$ in the linear regime due to the fact that the strain dependences of the two moduli are comparable at different polymer concentrations. This suggests that $\gamma_{X}$ follows the solid/ fluid balance as a function of polymer concentration in the linear regime. The frequency dependence of $\gamma_{X}$ also shows intermediate behavior, with the initial increase $(\omega$ $\leq 40 \mathrm{rad} / \mathrm{s})$ following the frequency dependence of $\gamma_{1}$ and the subsequent decrease $(\omega$ $>40 \mathrm{rad} / \mathrm{s}$ ) following the frequency dependence of $\gamma_{2}$.

We now consider the yield stresses at the three yield points: $\sigma_{1}, \sigma_{2}$, and $\sigma_{X}$ (Fig. 8). All three yield stresses show similar behavior, increasing with polymer concentration and frequency. The dependences on polymer concentration, i.e., strength of attraction, follow a power-law increase (log-log plots in the insets). Such a form has already been found for colloidal gels formed due to van der Waals attractions [Buscall et al. (1987); Larson (1999); Russel and Grant (2000)] and thermoreversible gels [Gopalakrishnan and Zukoski (2007)] for both increasing polymer and colloid concentrations. Furthermore, for 
depletion gels similar to ours, a power-law dependence has been predicted by an activated barrier hopping approach [Kobelev and Schweizer (2005a)]. This model is based on a shear-induced deformation and reduction of the free-energy barrier, which favors the escape of bonded particles. Adapting this approach to our system with its intercluster and intracluster bonds, our particles can be considered as trapped in a free-energy landscape involving two barriers. At the first yield point, intercluster bonds are broken, thus disrupting the gel network structure. The first yield stress $\sigma_{1}$ is thus expected to increase with attraction strength controlled by the polymer concentration. A fit of a power law $\sigma=\hat{\sigma}\left[\left(c_{p} / c_{p}^{\mathrm{gel}}\right)^{\mu}-1\right][$ Kobelev and Schweizer (2005a) $]$ to the data results in good agreement [Fig. 8(a)]. The obtained exponent $\mu=1.6$ is lower than experimentally determined values for similar systems [Buscall et al. (1987); Gopalakrishnan and Zukoski (2007); Larson (1999); Russel and Grant (2003)] and theoretical predictions [Kobelev and Schweizer (2005a)], which suggest values of $2.0 \leq \mu \leq 2.5$. Note that in our system experimental evidence exists [Dibble et al. (2006); Smith (2004)] that the number of bonds per particle decreases as a function of $c_{p} / c_{p}^{*}$, in contrast to the assumption used in theoretical calculations [Kobelev and Schweizer (2005a)]. This can be attributed to the fact that the theory is based on homogeneous gels while our gels, possibly as a result of a nonequilibrium route to gelation, present a large degree of heterogeneity. Such an additional dependence could be a reason for the smaller exponents found in our case. The position of the gel boundary derived from the power-law fit, $c_{p}^{\text {gel }}$, is found to be in the range $0.25 c_{p}^{*}<c_{p}^{\text {gel }}<0.32 c_{p}^{*}$. It has been fixed to $c_{p} / c_{p}^{*}=0.25$, which is consistent with our previous work [Laurati et al. (2009)] and leads to good fits at all frequencies. Fitted values of the prefactor $\hat{\sigma}$ increase with increasing frequency over the range $2 \leq \hat{\sigma}$ $\leq 5 \mathrm{~Pa}$. The agreement between the experimental data and the power-law scaling proposed by Kobelev and Schweizer (2005a) supports the interpretation of the first yield stress $\sigma_{1}$ as the one necessary to overcome the free-energy barrier localizing each particle, which is also consistent with the concept of bond breaking.

The incomplete yielding of samples at the first yield point suggests that particles are still trapped within clusters, which-however-can flow past each other, representing a cluster liquid. We propose that the second yield stress corresponds to a further disruption of these clusters under larger deformations. While the theory of Kobelev and Schweizer (2005a, 2005b) in its present form does not describe such a process, we speculate that in analogy to the first yield point, it might be described as a shear-induced reduction of entropic intracluster barriers where clusters are broken down to individual particles or smaller clusters allowing for a free flow. Again, this barrier increases with increasing polymer concentration, i.e., attraction strength. The second yield stress also follows a power-law dependence on $c_{p} / c_{p}^{*}$ with a slightly smaller exponent $1.2 \leq \mu \leq 1.4$. However, the agreement is not as good as for the first yield point. It is interesting to note that in this case the prefactor $\hat{\sigma}$ ranges from $10 \mathrm{~Pa}$ at $\omega=1 \mathrm{rad} / \mathrm{s}$ to $30 \mathrm{~Pa}$ at $\omega=100 \mathrm{rad} / \mathrm{s}$. These considerably different levels of stress characterizing the first and second yielding processes indicate a clear distinction between intracluster bond breaking and intercluster rearrangements. In addition, the average number of bonds in similar samples has been observed by confocal microscopy to decrease from 6 at $c_{p} / c_{p}^{*}=0.4$ to 4 at $c_{p} / c_{p}^{*}=0.8$ and further to 2 at $c_{p} / c_{p}^{*}=1.5$ [Smith (2004)]. The ratio $\sigma_{2} / \sigma_{1}$ shows a comparable dependence, thus reflecting the fact that, while the increase of the strength of bonds with increasing $c_{p} / c_{p}^{*}$ contributes to the increase of $\sigma_{2}$ (as for $\sigma_{1}$ ), less compact elongated clusters formed at larger polymer concentrations would need to break less bonds to rearrange, hence contributing to a relative decrease of $\sigma_{2}$. Finally, the stress at the crossing point, $\sigma_{X}$, also follows a power law with $\mu=1.9$ and $3 \leq \hat{\sigma} \leq 6 \mathrm{~Pa}$, as a result of being intermediate between the first and second yield points. 
All three yield stresses increase in value with increasing frequency (Fig. 8), which is reflected in the increasing $\hat{\sigma}$, as mentioned above. The same frequency dependence is observed in the linear regime [Laurati et al. (2009)], which suggests that in the case of the first yielding point the elastic strength of the samples in the quiescent state governs the stress needed to break the structure and fluidize the sample. In terms of the free-energy picture described above, the increase as a function of frequency of the prefactor used to fit the power-law dependence of stress on polymer concentration indicates an increase of the barriers with increasing frequency.

\section{Step rate tests}

In step rate tests, a constant rate of deformation is applied, the shear rate $\dot{\gamma}$, and the stress $\sigma$ measured as a function of time $t$ (Fig. 9). With strain $\gamma=\dot{\gamma} t$, the stress $\sigma$ initially increases linearly and then overshoots before it seems to tend toward a constant steadystate stress (within the measurement time of $1000 \mathrm{~s}$ a well-developed steady-state could only be observed for the largest shear rates). Two distinct stress overshoots are observed for polymer concentrations $c_{p} / c_{p}^{*} \leq 1$, whereas for larger polymer concentrations, $c_{p} / c_{p}^{*}$ $=2.0[$ Fig. $9(\mathrm{~d})]$, only the second stress overshoot is clearly visible, while the first stress overshoot is reduced to a shoulder. With increasing polymer concentration, the strain at the first overshoot or shoulder, $\gamma_{1}^{\text {step }}$, is initially constant with $\gamma_{1}^{\text {step }} \approx 12 \%$ for $c_{p} / c_{p}^{*}<1$ and then increases to about $20 \%$ for $c_{p} / c_{p}^{*}=1.5$ [Fig. 10(a)]. The corresponding stress at the first overshoot, $\sigma_{1}^{\text {step }}$, increases with polymer concentration [Fig. 10(a), inset]. The second stress overshoot moves to smaller strains upon increasing the polymer concentration; $\gamma_{2}^{\text {step }}$ decays from about $300 \%$ to $150 \%$ [Fig. 10(b)]. The corresponding stress at the second overshoot, $\sigma_{2}^{\text {step }}$, increases with polymer concentration [Fig. 10(b), inset]. The shear rate $\dot{\gamma}$ has only a weak effect on the overshoots; increasing $\dot{\gamma}$ slightly shifts the first overshoot to higher strains and the second overshoot to lower strains (arrows in Fig. 9).

Two stress overshoots are consistent with the presence of two yielding processes, as found in DSS (Sec. III D 1). A stress overshoot in step rate tests has also been reported for other systems such as polymer melts [Osaki et al. (2000)], nanocomposite materials [Letwimolnun et al. (2007)], metallic glasses [van Aken et al. (2000)], and concentrated colloidal suspensions with both repulsive sphere and attractive interactions [Carrier and Petekidis (2009); Zausch et al. (2008)]. MCT relates the stress overshoot seen in suspensions of hard spheres to superdiffusive motion of the particles, which has been observed in the transient dynamics of this system [Zausch et al. (2008)]. Similarly, simulations relate the overshoot to a distortion of the structure, characterized by an anisotropic component of the pair correlation function [Zausch and Horbach (2009)]. While only one stress overshoot has been found in hard-sphere systems, two have been observed in attractive glasses [Pham et al. (2008)].

The strain at which the first stress overshoot appears, $\gamma_{1}^{\text {step }}$, is slightly larger than, but still comparable to, the first yield strain observed in DSS, $\gamma_{1}$, and furthermore shows a similar dependence on polymer concentration [Fig. 7(a)]. A power-law fit to the stress $\left(\sigma_{1}^{\text {step }}\right)$ [Kobelev and Schweizer $\left.(2005 a)\right]$ yields good agreement with the data and an exponent $\mu=1.7$, consistent with the findings of the DSS experiments (Sec. III D 1) and other studies [Buscall et al. (1987); Gopalakrishnan and Zukoski (2007); Kobelev and Schweizer (2005a); Larson (1999); Russel and Grant (2000)]. The prefactor of the powerlaw fit, $1 \leqq \hat{\sigma} \lesssim 2 \mathrm{~Pa}$, is also in agreement with values obtained for $\sigma_{1}$ from DSS measurements. This suggests that the first stress overshoot is also related to the breaking of intercluster bonds. Shear induces a stretching of the bonds, which represents an elastic deformation with the buildup of stress until the bonds break at the first yield strain $\left(\gamma_{1}^{\text {step }}\right)$. 


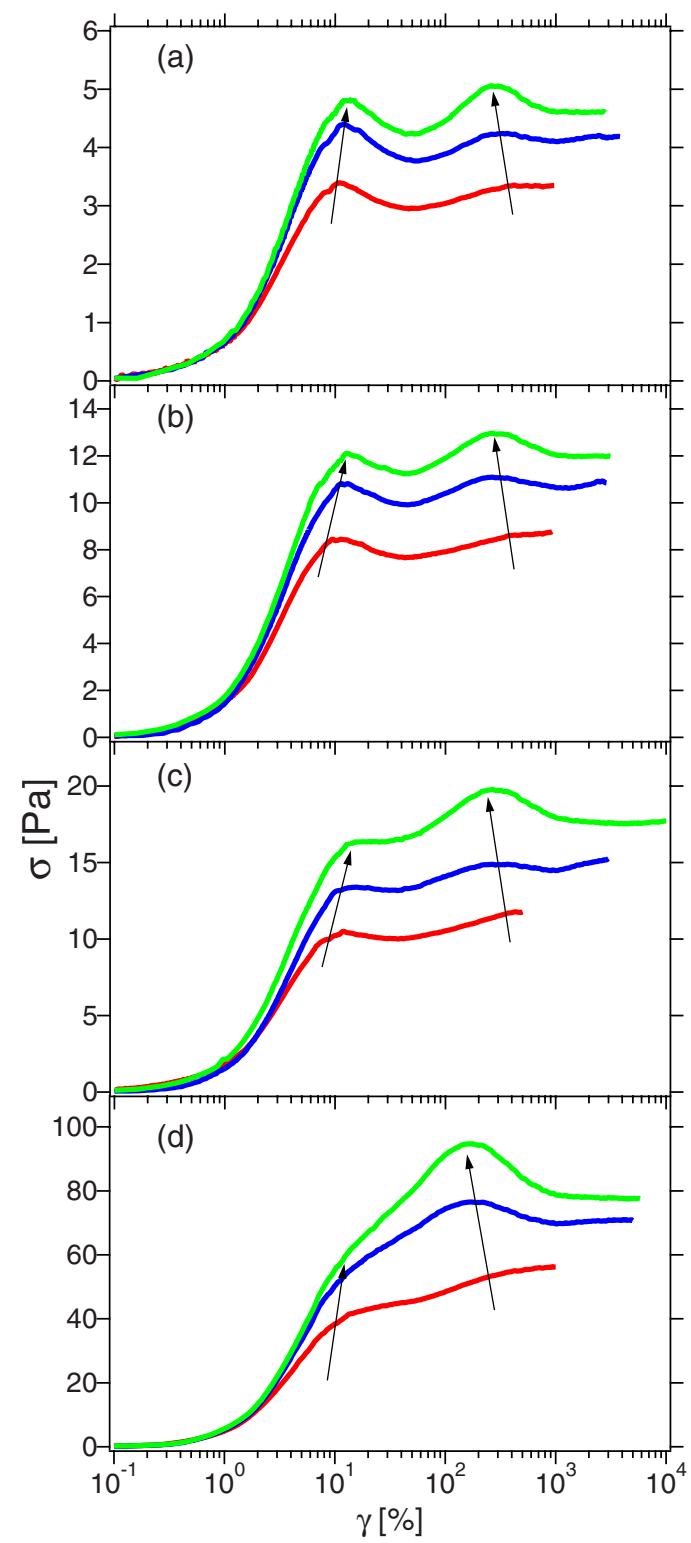

FIG. 9. Step rate tests with stress $\sigma$ as a function of strain $\gamma=\dot{\gamma} t$ for samples with polymer concentrations $c_{p} / c_{p}^{*}=0.5(\mathrm{a}), 0.7(\mathrm{~b}), 1.0(\mathrm{c})$, and 2.0 (d) and shear rates (increasing from bottom to top at high strains) $\dot{\gamma}$ $=0.01,0.05,0.10 \mathrm{~s}^{-1}[(\mathrm{a}),(\mathrm{b}),(\mathrm{d})]$ and $0.005,0.03,0.10 \mathrm{~s}^{-1}$ (c). The duration of the experiments was $t$ $=1000 \mathrm{~s}$. Arrows indicate yielding points and point in the direction of increasing shear rate.

The balance between the decreasing range and the increasing strength of the bonds is again consistent with the dependence of the yield strain on polymer concentration (Sec. IV). The increase of $\gamma_{1}^{\text {step }}$ with increasing shear rate is also supportive of the findings from the DSS experiments.

The second stress overshoot at larger strains, $\gamma_{2}^{\text {step }}[$ Fig. 10(b)], supports the existence of a second yielding process which involves the breaking of intracluster bonds along with the deformation of clusters. Again, the value of $\gamma_{2}^{\text {step }}$ as well as its dependence on polymer 


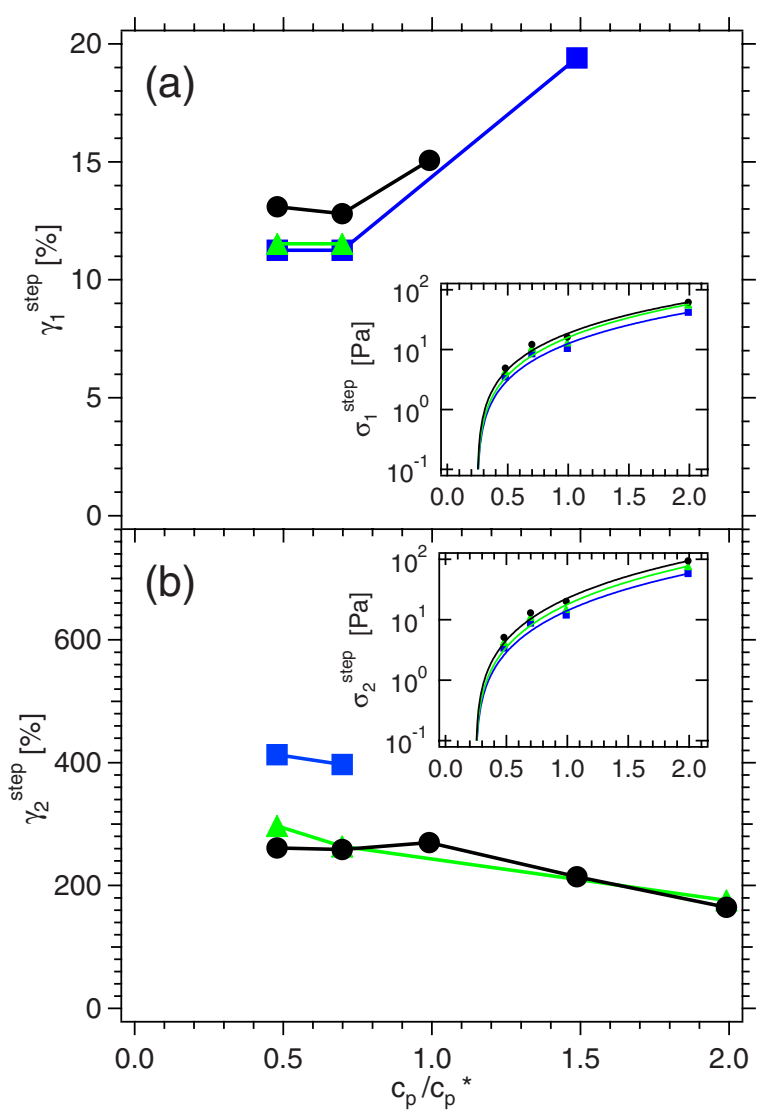

FIG. 10. Strains at (a) the first overshoot or shoulder, $\gamma_{1}^{\text {step }}$, and (b) the second overshoot, $\gamma_{2}^{\text {step }}$, as a function of polymer concentration $c_{p} / c_{p}^{*}$ for different shear rates $\dot{\gamma}=0.01 \mathrm{~s}^{-1}(\boldsymbol{\square}), 0.05 \mathrm{~s}^{-1}(\mathbf{\Delta})$, and $0.10 \mathrm{~s}^{-1}(\boldsymbol{\bullet})$. The insets show the corresponding stresses, $\sigma_{1}^{\text {step }}$ and $\sigma_{2}^{\text {step }}$, for the same shear rates with corresponding power-law fits. The strains and stresses have been extracted from step rate tests.

concentration and shear rate resembles that of the second yield strain $\gamma_{2}$ observed in DSS experiments [Fig. 7(c)], where the decreases of $\gamma_{2}$ with polymer concentration and shear rate have been attributed to the decreasing cluster size (Sec. III D 1). The corresponding stress again shows a power-law dependence on $c_{p} / c_{p}^{*}$ with an exponent $\mu=2.0$. The values of the stresses at the second yield point are very similar to those of the first yield point with a similar prefactor $\hat{\sigma} \approx 2 \mathrm{~Pa}$, which is an order of magnitude lower than the corresponding values from DSS measurements.

To further investigate the relation between the overshoots in the step rate tests and the yield points in the DSS experiments, the stress-strain data from the step rate tests (Fig. 9) are regrouped; the results for different polymer concentrations $c_{p} / c_{p}^{*}$ but a fixed shear rate $\dot{\gamma}=0.10 \mathrm{~s}^{-1}$ are collected and shown in Fig. 11. This representation reveals the similarity to the strain dependence of the elastic stress $G^{\prime} \gamma$ as determined in a DSS experiment [Fig. 6(b)]. In both cases, the strain dependence shows two maxima or shoulders before a steady-state value is attained. With increasing polymer concentration, the first maximum evolves into a shoulder and both maxima (or shoulders) move closer, i.e., the strains of the first maxima, $\gamma_{1}$ and $\gamma_{1}^{\text {step }}$, increase and the strains of the second maxima, $\gamma_{2}$ and $\gamma_{2}^{\text {step }}$, decrease. The steady-state values of the stress $\sigma_{\infty}^{\text {step }}$ increase with polymer concentration and rate $\dot{\gamma}$ [Fig. 12(b), full symbols]. 


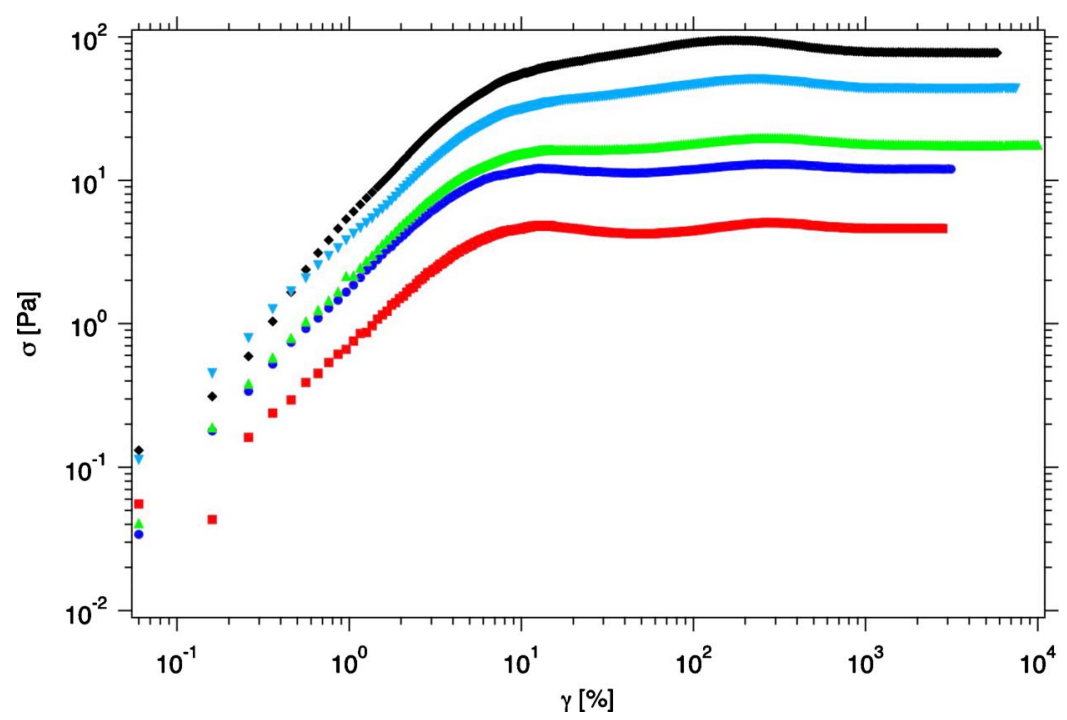

FIG. 11. Step rate tests with stress $\sigma$ as a function of strain $\gamma=\dot{\gamma} t$ at a shear rate $\dot{\gamma}=0.10 \mathrm{~s}^{-1}$ for samples with polymer concentrations $c_{p} / c_{p}^{*}=0.5,0.7,1.0,1.5$, and 2.0 (from bottom to top).

These steady-state values of the stress $\sigma_{\infty}^{\text {step }}$ are compared to flow curves, i.e., the stress $\sigma$ as a function of shear rate $\dot{\gamma}$, as determined in a steady rate sweep experiment performed upon decreasing rate [Fig. 12(a)]; the data essentially agree (the stress is, particularly at low rates, slightly underestimated in the standard steady rate sweeps, which we attribute to the long relaxation time needed to reach the steady-state). The flow curves do not exhibit a clear yield-stress plateau. This could be due to the fact that measurements were conducted upon decreasing shear rate starting from high rates where the network structure is broken. Thus, at low rates a long time would be needed for network formation. Furthermore, the "plateau" has a finite slope in step rate experiments, especially for $c_{p} / c_{p}^{*}=1.0$, where a very small shear rate was measured. This is consistent with activated hopping processes leading to bond breaking, and thus a plateau is not expected to develop [Kobelev and Schweizer (2005b)]. The experimental data in the lowest 2 decades exhibit a smooth power-law dependence on shear rate with an exponent of about 0.23 , in agreement with such theoretical predictions. We can extract an indicative yield stress from the value at the lowest shear rate measured, as a function of polymer concentration. Again, the yield stress $\sigma_{0}$ follows a power-law dependence [Kobelev and Schweizer (2005a)] with an exponent $\mu=1.9$, consistent with the findings of other tests (Sec. III D 1) as well as other studies [Buscall et al. (1987); Gopalakrishnan and Zukoski (2007); Kobelev and Schweizer (2005a); Larson (1999); Russel and Grant (2000)].

\section{Step stress and recovery tests}

In a step stress (creep) test, a step of stress $\sigma$ is applied and the induced strain $\gamma(t)$ is measured as a function of time $t$ (Fig. 13). After the initial oscillations due to coupling of the tool inertia with the viscoelastic properties of the sample, the strain $\gamma(t)$ increases sublinearly as a function of time provided the applied stress $\sigma$ is well below the first yield stress $\sigma_{1}\left[\sigma_{1}=3 \mathrm{~Pa}\right.$ for $c_{p} / c_{p}^{*}=0.5$ and $\sigma_{0}=10 \mathrm{~Pa}$ for $c_{p} / c_{p}^{*}=1.0$ at $\omega=1 \mathrm{rad} / \mathrm{s}$, from DSS experiments; Fig. 8(a)]. The strain $\gamma(t)$ does not attain a constant value as would be expected for an ideal elastic solid [Figs. 13(a) and 13(c)], i.e., we observe a so-called 

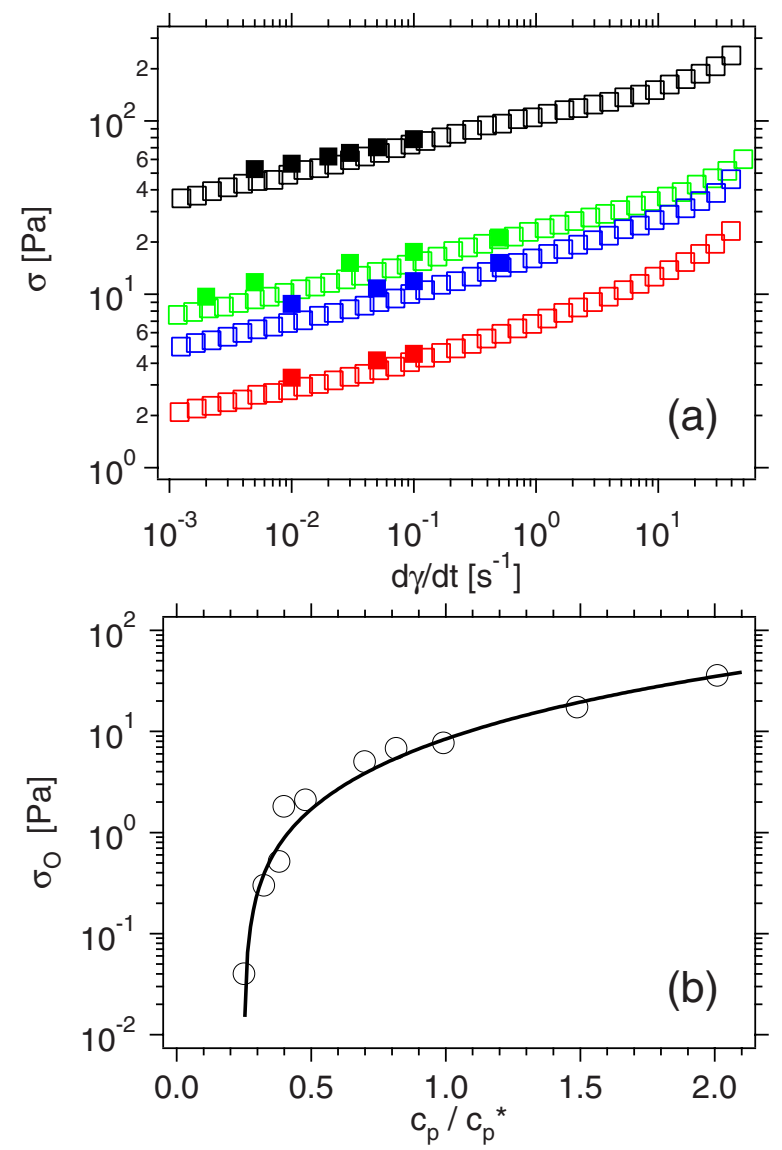

FIG. 12. (a) Stress $\sigma$ as a function of shear rate $\dot{\gamma}$ (flow curve) as determined in a standard measurement (open symbols) and the steady-state value $\sigma_{\infty}^{\text {step }}$ from step rate tests (full symbols) for different polymer concentrations $c_{p} / c_{p}^{*}=0.5,0.7,1.0$, and 2.0 (from bottom to top). (b) Stress $\sigma_{0}$, corresponding to the stress measured at the lowest rate in steady rate sweeps, as a function of polymer concentration. The line is a fit to the power-law dependence $\sigma=\hat{\sigma}\left[\left(c_{p} / c_{p}^{\mathrm{gel}}\right)^{\mu}-1\right]$.

solid creep response. Correspondingly, the viscosity $\eta(t)$ increases with time [Fig. 13(b), $\sigma=1 \mathrm{~Pa}$, and Fig. 13(d), $\sigma=2$ and $8 \mathrm{~Pa}$. If the applied stress $\sigma$ becomes comparable or larger than the first yield stress $\sigma_{1}$, a complex response is observed as a function of time, with regions of decreasing viscosity (shear thinning, $\gamma \sim t^{v}$, with $v>1$ ) alternating with regions of increasing viscosity (shear thickening or creeping, $\gamma \sim t^{v}$, with $v<1$ ), before the system reaches a constant viscosity (steady-state flow, $\gamma \sim t$ ). For values of the stress significantly larger than $\sigma_{1}$ but still lower than $\sigma_{2}$, the viscosity shows a maximum as a result of the transition from shear thickening to shear thinning [Figs. 13(b) and 13(d)]. Such a maximum, or equivalently the onset of shear thinning, shifts toward shorter times with increasing stress until, for $\sigma>\sigma_{2}$, shear thinning is observed immediately after application of stress.

The complex response upon applying a stress $\sigma$ with $\sigma_{1}<\sigma<\sigma_{2}$ reflects the presence of two yielding processes and is reminiscent of the behavior of attractive glasses [Pham et al. (2008)]. As the strain increases with time, the samples pass through regimes with intercluster and intracluster bond breaking as well as bond reformation.

To investigate the transition from creep to flow behavior upon increasing the applied 


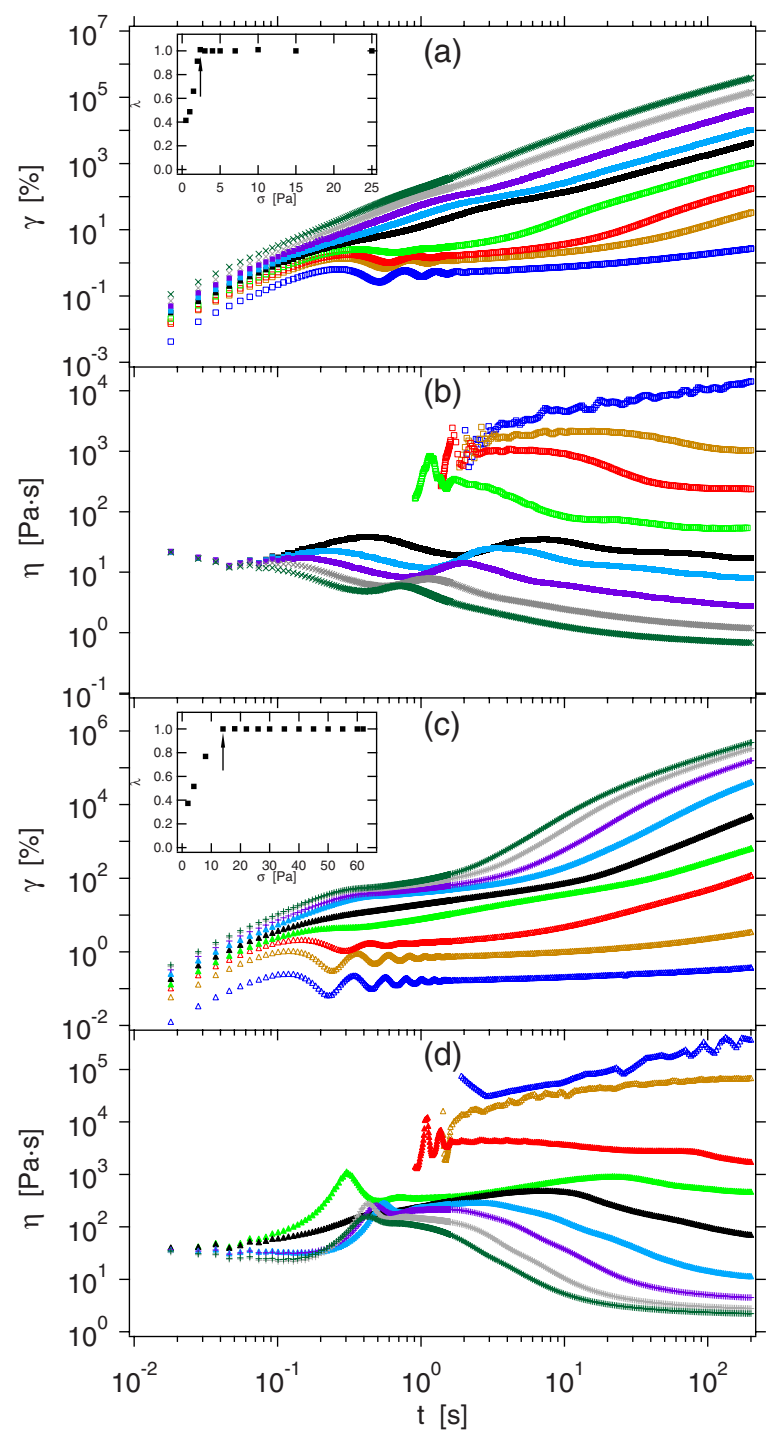

FIG. 13. (a),(c) Strain $\gamma$ and (b),(d) viscosity $\eta$ as functions of time $t$ as determined in step stress (creep) tests for samples with polymer concentrations $c_{p} / c_{p}^{*}=0.5[(\mathrm{a}),(\mathrm{b})]$ and $1.0[(\mathrm{c}),(\mathrm{d})]$ for different applied stresses $\sigma$ $=1.0,2.0,2.4,3.0,4.0,5.0,7.0,10.0$, and 15.0 Pa [(a) from bottom to top; (b) from top to bottom] and 2.0, 8.0, $14.0,18.0,26.0,35.0,45.0,55.0$, and $62.0 \mathrm{~Pa}$ [(c) from bottom to top; (d) from top to bottom]. According to the DSS experiments, these stresses are below the first yield stress, $\sigma<\sigma_{1}$ (open symbols); between first and second yield stresses, $\sigma_{1}<\sigma<\sigma_{2}$ (full symbols); and beyond the second yield stress, $\sigma>\sigma_{2}$ (crosses and plus). Insets: Creep exponent $\lambda$ as a function of stress $\sigma$ for the same samples. Arrows indicate when the plateaux $\lambda \approx 1$ are reached.

stress $\sigma$, a power-law dependence $\gamma(t) \sim t^{\lambda}$ was fitted to the data at long times, $150<t$ $<200 \mathrm{~s}$, although this is only a limited region. The strain dependence of the creep exponent $\lambda$ provides the transition from creep $(\lambda<1)$ to flow $(\lambda \approx 1)$, which occurs at the yield stress $\sigma_{\lambda}$ (Fig. 13, arrows in insets). The values of $\sigma_{\lambda}$ (Fig. 14) and their dependence on polymer concentration are consistent with the values of the first yield stress obtained from DSS, $\sigma_{1}$ [Fig. 8(a)], and the first and second yield stresses obtained from step rate tests, $\sigma_{1}^{\text {step }}$ and $\sigma_{2}^{\text {step }}$ (Fig. 10, insets). The dependence of $\sigma_{\lambda}$ on polymer concentration 


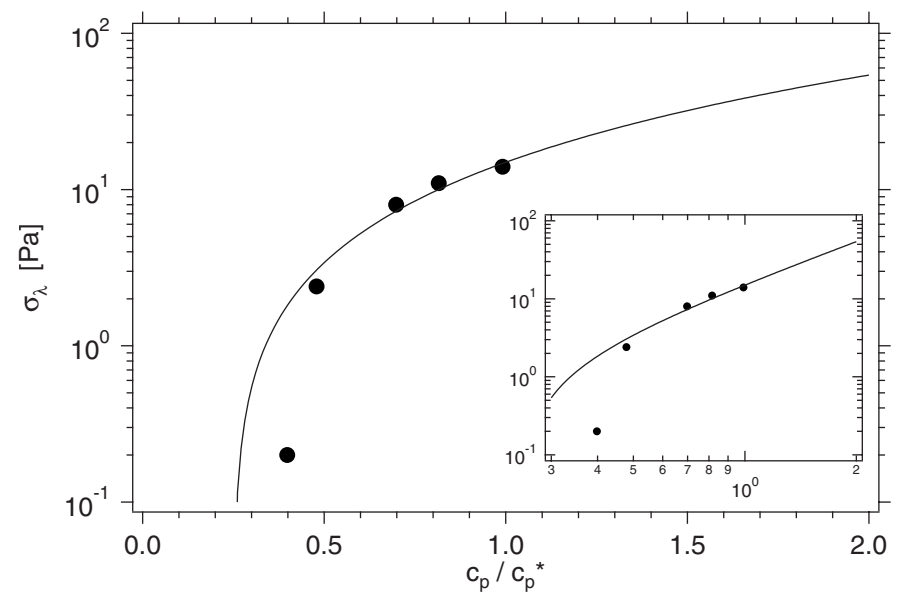

FIG. 14. Stress $\sigma_{\lambda}$ at the transition from creep to flow as a function of polymer concentration $c_{p} / c_{p}^{*}$ as extracted from step stress (creep) tests. The solid line represents a power-law fit. Inset: Log-log plot of the same data.

also agrees with a power law [Kobelev and Schweizer (2005a)] with an exponent $\mu$ $=1.8$ (Fig. 8), which is consistent with the results of our DSS and step rate experiments (Secs. III D 1 and III D 2) and other studies [Buscall et al. (1987); Gopalakrishnan and Zukoski (2007); Kobelev and Schweizer (2005a); Larson (1999); Russel and Grant (2000)].

When the applied stress $\sigma$ is removed after a step stress experiment, the system relaxes. This relaxation was followed and the recovered strain $\gamma_{r}(t)$ was measured over time $t$, where $\gamma_{r}(t)=\gamma_{\max }-\gamma(t)$, with $\gamma_{\max }$ being the maximum strain reached at the end of the application of stress [Figs. 15(a) and 15(b)]. A part of the strain recovers almost immediately within the fist second. This is followed by a slow relaxation to a steadystate, the maximum recovered strain $\gamma_{r}^{\max }$, within 100-400 s, depending on the applied stress $\sigma$. The maximum recovered strain $\gamma_{r}^{\max }$ first increases with increasing applied stress $\sigma$ until it reaches a maximum, $\gamma_{1}^{\text {creep }}$ at $\sigma_{1}^{\text {creep }}$, and then decreases to a steady-state value $\gamma_{2}^{\text {creep }}$, which is attained at $\sigma_{2}^{\text {creep }}$ and only clearly detected at lower $c_{p} / c_{p}^{*}$, probably due to the lower absolute value of the yield stresses [Fig. 15(c)]. With increasing polymer concentration, the maximum $\gamma_{1}^{\text {creep }}$ first grows from $c_{p} / c_{p}^{*}=0.4$ to 0.5 , then decreases at $c_{p} / c_{p}^{*}=0.7$, and successively increases again at larger polymer concentrations [Fig. 16(a)]. At the same time, the position of the maximum, $\sigma_{1}^{\text {creep }}$, increases with polymer concentration [Fig. 16(a), inset]. The plateau $\gamma_{2}^{\text {creep }}$ decreases with increasing polymer concentration, and its beginning shifts to larger stresses $\sigma_{2}^{\text {creep }}$ [Fig. 16(b)].

In all cases, the maximum recovered strain $\gamma_{r}^{\max }$ is much smaller than the maximum strain reached at the end of the application of stress, $\gamma_{\max }$ [Fig. 15(c)]. At small applied stresses, the sample elastically relaxes with the recovered strain $\gamma_{r}$ proportional to the applied stress $\sigma$. An almost elastic recovery is observed up to the maximum recovered strain $\gamma_{1}^{\text {creep }}$ at $\sigma_{1}^{\text {creep }}$, which is in agreement with the transition from creep to flow observed upon application of stress, $\sigma_{1}^{\text {creep }} \approx \sigma_{\lambda}$ (Fig. 14). At the same stress $\sigma_{1}^{\text {creep }} \approx \sigma_{\lambda}$, i.e., at the end of creep and the beginning of flow, plastic deformation starts [Fig. 15(c), inset]. In the plastic regime, the sample recovers only a very small fraction of its deformation. This is consistent with increasing structural disruption of the particle network and the presence of independent clusters or, at higher stresses, individual particles. Interestingly, the values of $\gamma_{r}^{\max }$ at $c_{p} / c_{p}^{*}=0.5$ and 1.0 coincide with the onset of structural and dynamic 

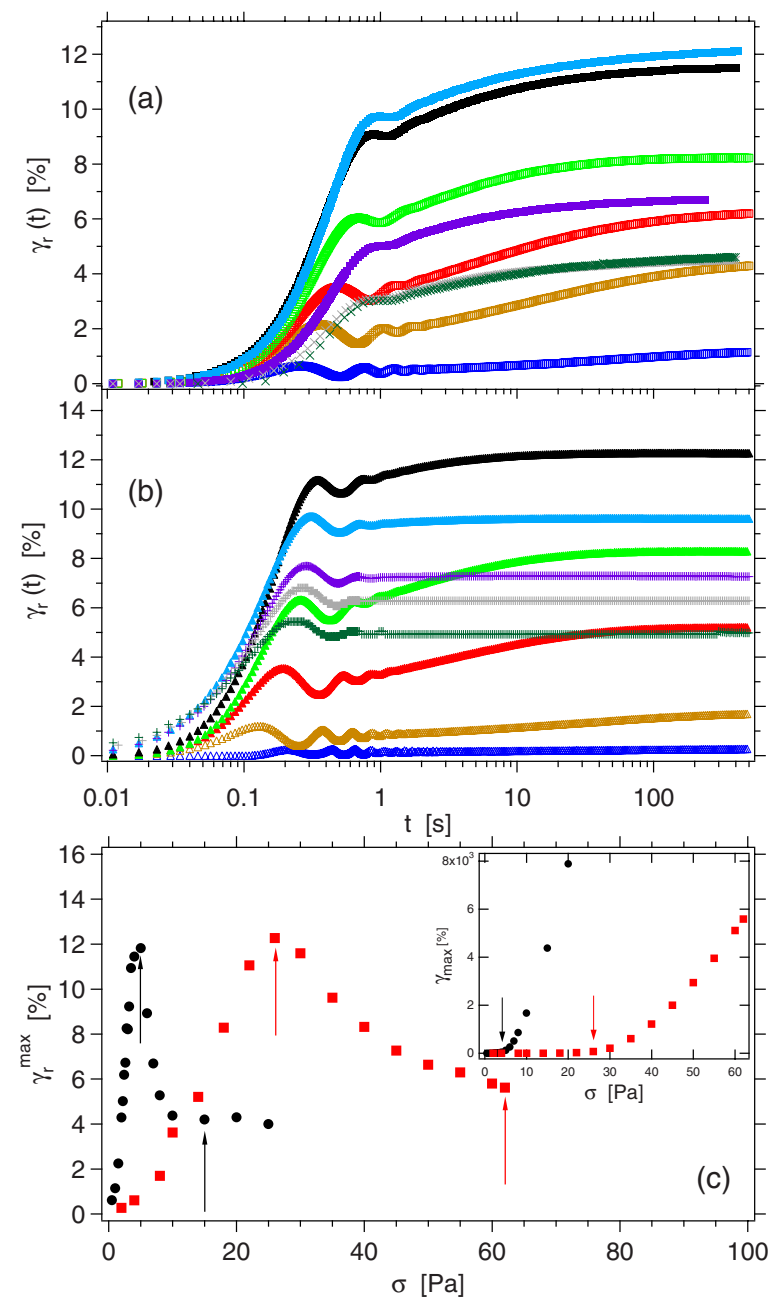

FIG. 15. (a),(b) Recovered strain $\gamma_{r}$ as a function of time $t$ and (c) maximum recovered strain $\gamma_{r}^{\max }$ as a function of applied stress $\sigma$. Both are from the relaxation after step stress (creep) experiments for samples with polymer concentrations $c_{p} / c_{p}^{*}=0.5[(\mathrm{a}) ;-$ in (c)] and $1.0[(\mathrm{~b}) ; \boldsymbol{\square}$ in (c)] for different applied stresses $\sigma=1.0,2.0,2.4$, 3.0, 4.0, 5.0, 7.0, 10.0, and 15.0 Pa (a) and 2.0, 8.0, 14.0, 18.0, 26.0, 35.0, 45.0, 55.0, and 62.0 Pa (b), with symbols as in Fig. 13. Arrows in (c) indicate the maxima of $\gamma_{r}^{\max }$ and the beginning of the plateaux. Inset: Maximum strain at the end of the step stress as a function of applied stress. Arrows indicate onset of yielding.

changes observed in confocal microscopy and scattering experiments of very similar samples [Smith (2004); Smith et al. (2007)]. The stress $\sigma_{1}^{\text {creep }}$ and strain $\gamma_{1}^{\text {creep }}$ at the maximum are expected to be affected by both cluster and bond elasticity. At the end of stress application, the network structure seems to have been broken (they creep), but nevertheless no simple flow is observed, indicating that some structure (clusters) is present. This is similar to what was observed for the crossing point of $G^{\prime}$ and $G^{\prime \prime}$. The values of $\gamma_{1}^{\text {creep }}$ and $\sigma_{1}^{\text {creep }}$ are indeed similar to those of $\gamma_{X}$ and $\sigma_{X}$ observed in DSS experiments [Figs. 7(b) and 8(b)]. An effect of the cluster size is suggested by the dependence on polymer concentration; $\gamma_{1}^{\text {creep }}$ shows a minimum that coincides with the maximum size of structural heterogeneities (or clusters) at this polymer concentration [Laurati et al. (2009)]. Furthermore, with increasing polymer concentration $c_{p} / c_{p}^{*}$ the 


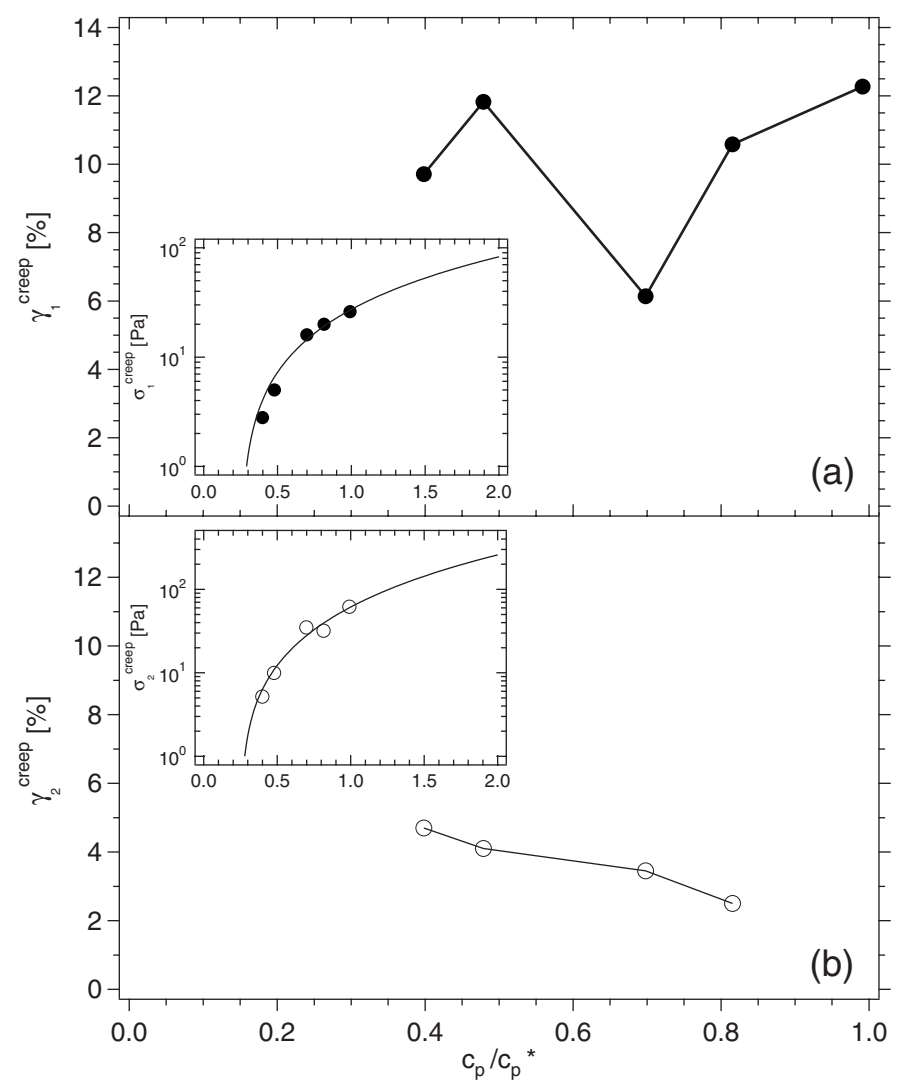

FIG. 16. Strains and stresses (insets) at (a) the maximum, $\gamma_{1}^{\text {creep }}$ and $\sigma_{1}^{\text {creep }}$, and (b) the beginning of the plateau, $\gamma_{2}^{\text {creep }}$ and $\sigma_{2}^{\text {creep }}$, of the maximum recovered strain, $\gamma_{\mathrm{r}}^{\max }(\sigma)$ [arrows in Fig. 15(c)] as functions of polymer concentration $c_{p} / c_{p}^{*}$, as extracted from recovery experiments after step stress (creep) tests.

attraction strength increases, which is consistent with the observed increase of $\sigma_{1}^{\text {creep }}$ with polymer concentration [Fig. 16(a), inset]. The predicted power-law dependence [Kobelev and Schweizer (2005a)] again shows good agreement and results in an exponent $\mu$ $=1.8$, consistent with the results of our DSS, step rate, and step stress experiments (Secs. III D 1 and III D 2 and above) and other reports [Buscall et al. (1987); Gopalakrishnan and Zukoski (2007); Kobelev and Schweizer (2005a); Larson (1999); Russel and Grant (2000)].

At very high stresses, beyond $\sigma_{2}^{\text {creep }}$, the recovered strain saturates at $\gamma_{2}^{\text {creep }}$. This residual elastic response suggests that some structures, small clusters, or heterogeneities remain or are reformed, depending on the strength and range of the attractions. The steady-state strain $\gamma_{2}^{\text {creep }}$ decreases with increasing polymer concentration [Fig. 16(b)], reflecting the decreasing effective range of the potential $\xi^{*}$ (Table I). Its value in addition is similar to the effective range; $\gamma_{2}^{\text {creep }} \approx \xi^{*}$. The value of $\sigma_{2}^{\text {creep }}$ is related to the force required to break down clusters or to overcome a second free-energy barrier localizing particles within clusters. This is consistent with the power-law increase of $\sigma_{2}^{\text {creep }}$ with polymer concentration $c_{\mathrm{p}} / c_{\mathrm{p}}^{*}$ and thus attraction strength with an exponent $\mu=2.0$. Furthermore, the values of $\sigma_{2}^{\text {creep }}$ and $\sigma_{2}$ extracted from DSS experiments [Fig. 8(c)] are found to be in agreement. 


\section{QUANTITATIVE MODELS FOR THE FIRST YIELD POINT}

The results of our rheological experiments suggest that the yielding of gels is closely linked to "bond breaking," i.e., the escape of particles from the attractive potential wells of their neighbors. We recently proposed a model for the escape of a particle from the attractive well of one neighbor [Lekkerkerker et al. (1992); Smith et al. (2007)]. Although this represents a simplified two-particle situation, it showed semiquantitative agreement with the observed bond lifetimes in samples with different polymer concentrations [Laurati et al. (2009)] and the shear-induced crystallization boundary found under oscillatory shear [Smith et al. (2007)]. This suggests that breaking one individual bond already allows the particle to significantly rearrange.

We only briefly summarize the model [Laurati et al. (2009); Smith et al. (2007)]. The model is based on Kramers' approach to estimate the escape time $\tau$ of a particle from an attractive potential well [Kramers (1940)]. The first passage time of a Brownian particle in an AO potential can be calculated numerically [McLeish (2000)]. However, to obtain an analytical expression, we approximate the potential by a ramp potential with the same depth $U_{0}$ and width $\delta^{*}=2 \xi^{*} R$ as the AO potential, where both $U_{0}$ and $\xi^{*}$ are estimated according to the GFVT [Aarts et al. (2002); Fleer and Tuinier (2007); Lekkerkerker et al. (1992)] (Table I). The escape time from this ramp potential is [McLeish (2000); Smith et al. (2007)]:

$$
\tau=\frac{1}{D_{\mathrm{S}}} \int_{0}^{\delta^{*}} \mathrm{~d} x^{\prime} e^{\beta U\left(x^{\prime}\right)} \int_{-\infty}^{x^{\prime}} \mathrm{d} x e^{-\beta U(x)}=\frac{\delta^{* 2}}{D_{\mathrm{S}}} \frac{e^{-\beta U_{0}}-\left(1-\beta U_{0}\right)}{\left(\beta U_{0}\right)^{2}},
$$

where $1 / \beta=k_{B} T$ is the thermal energy and $D_{\mathrm{S}}$ the short-time self-diffusion coefficient of the particle inside the potential well. We approximate $D_{\mathrm{S}}$ by the value of a particle in a hard-sphere suspension with volume fraction $\phi=0.55$ reflecting the heterogeneous structure of the samples, $D_{\mathrm{S}}=0.15 D_{0}$, with $D_{0}$ being the diffusion coefficient of an individual particle in a dilute solution [Banchio and Brady (2003)].

We consider oscillatory shear as applied in DSS experiments (Sec. III D 1). The application of shear separates initially close particles if they are in different constantvelocity planes. The increased interparticle separation reduces the potential barrier, which the particle needs to overcome to free itself, and thus the escape time $\tau$ is also reduced. The escape time $\tau$ decreases with increasing strain and also depends on the relative initial location of the two particles, since this determines the maximum displacement that can be induced by oscillatory shear [Smith et al. (2007)]. If $\tau$ becomes smaller than the residence time $\tau_{\mathrm{r}}$ in the displaced configuration, the particle is likely to escape the attractive well and the bond is broken. We consider a configuration to be displaced if its strain is larger than a threshold strain $\gamma^{\prime}$ and thus from geometrical considerations $\tau_{\mathrm{r}}\left(\gamma^{\prime}, \gamma\right)$ $=(2 / \omega) \arccos \left(\gamma^{\prime} / \gamma\right)$ with the strain amplitude $\gamma$. If the residence time $\tau_{\mathrm{r}}\left(\gamma^{\prime}, \gamma\right)$ is larger than the corresponding escape time $\tau\left(\gamma^{\prime}\right)$, the particle can escape the attractive potential. In the calculation of the escape time $\tau$, the attempt rate is dominated by Brownian motion, $D_{\mathrm{S}} / \delta^{* 2} \geq 290 \mathrm{~Hz}$, also under oscillatory shear, because the particles are small and the frequencies applied in our DSS experiments are low; $\omega / 2 \pi \lesssim 17 \mathrm{~Hz}$. (This is in contrast to the situation in [Smith et al. (2007)].) For each polymer concentration and frequency, we calculate $\tau\left(\gamma^{\prime} / \gamma\right)$ for different values of the parameter $\gamma$, and we find the optimum value of $\gamma$ (and $\gamma^{\prime} / \gamma$ ) for which the equation $\tau=\tau_{\mathrm{r}}$ first presents a root. For strains larger than this optimum value of $\gamma$, the particle is likely to escape the attractive well and thus break the bond since $\tau<\tau_{\mathrm{r}}$. This is considered as the first yield strain $\gamma_{1}$. Based on this kinetic criterion, we calculate $\gamma_{1}$ and its dependence on polymer concentration $c_{p} / c_{p}^{*}$ and frequency $\omega$ and compare these predictions to our data [Fig. 17]. The 


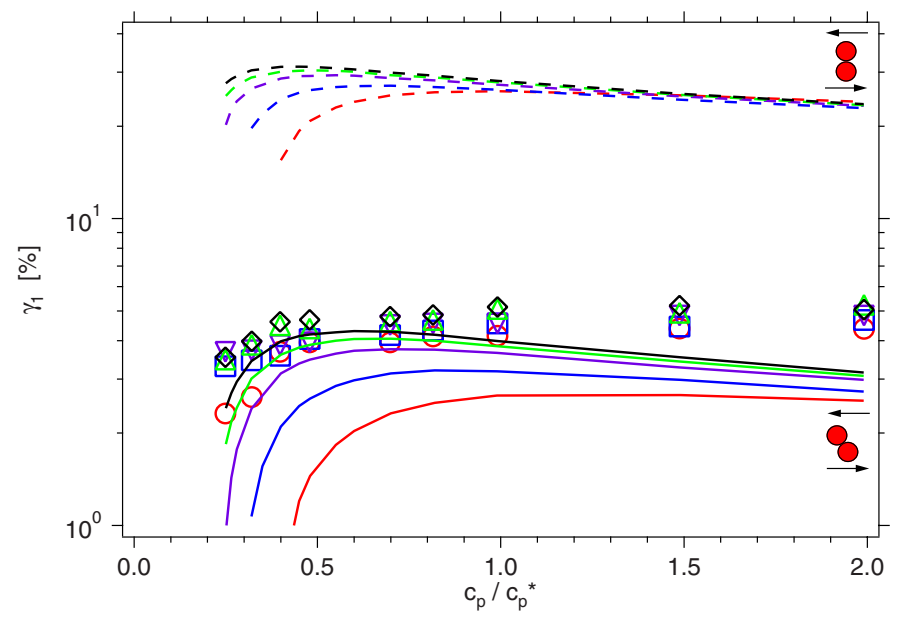

FIG. 17. First yield strain $\gamma_{1}$ as a function of polymer concentration $c_{p} / c_{p}^{*}$ extracted from DSSs as the onset of the sublinear stress-strain relation, with frequencies $\omega=1 \mathrm{rad} / \mathrm{s}(\bigcirc), 10 \mathrm{rad} / \mathrm{s}(\square), 40 \mathrm{rad} / \mathrm{s}(\nabla), 69 \mathrm{rad} / \mathrm{s}(\triangle)$, and $100 \mathrm{rad} / \mathrm{s}(\diamond)$. Lines represent predictions for the first yield strain $\gamma_{1}$ based on particles which are touching and whose relative initial location leads to the maximum-achievable separation (solid line) or is in the gradient direction (dashed line), as indicated. Colors correspond to the same frequencies as experimental data.

calculations have been performed based on two initial configurations: the particles located such that the maximum shear-induced separation is achieved (solid line) and the particles located along the gradient direction (dashed line, Fig. 17). The above imply a number of assumptions: Only one "bonded" neighbor (i.e., a total of two particles) is considered, neglecting the effect of multiple bonds to several neighbors and also ignoring the reformation of bonds. The effective colloid-colloid potential (bond) is furthermore approximated by an attractive ramp potential, commonly used at large polymer concentrations [Fuchs and Schweizer (2002)]. Finally, hydrodynamics are neglected.

In Fig. 17 we compare the yield strain $\gamma_{1}$ calculated at different frequencies to the corresponding experimental data extracted from DSSs. The model reproduces the qualitative dependence of the first yield strain $\gamma_{1}$ on polymer concentration $c_{p} / c_{p}^{*}$ with the two initial configurations bracketing the experimental data (Fig. 17). The most efficient configuration (solid line) represents one limit and, as expected, underestimates the yield strain $\gamma_{1}$. In contrast, particles arranged along the gradient direction are separated "tangentially" by shear, which is not efficient and thus overestimates the required yield strain (dashed line). The calculations, contrary to the experiments, show a small decrease in the yield strain at large polymer concentrations. A plateau or a slight decrease in $\gamma_{1}$ at large polymer concentrations results from a balance between the increased attraction strength $U_{0}$ and the decreased range $\xi^{*}$ (Table I). Our estimates of $\xi^{*}$ and $U_{0}$ were obtained in the limit of good solvent conditions, although our conditions approach those of a $\theta$ solvent, which might account for some of the discrepancy between our experiments and the model. The model also correctly reproduces the frequency dependence of $\gamma_{1}$ showing with increasing frequency. Moreover, the flatter dependence on polymer concentration observed experimentally with increasing frequency is also obtained through the model. Therefore, despite its simplicity, our model successfully predicts the main characteristics of the first yield strain $\gamma_{1}$ and thus captures the essential processes during the first yielding. Although the above model is only strictly applicable to DSS experiments, the first yield strains obtained by other methods are very similar and show a similar dependence on polymer concentration, which is again semiquantitatively captured by our 

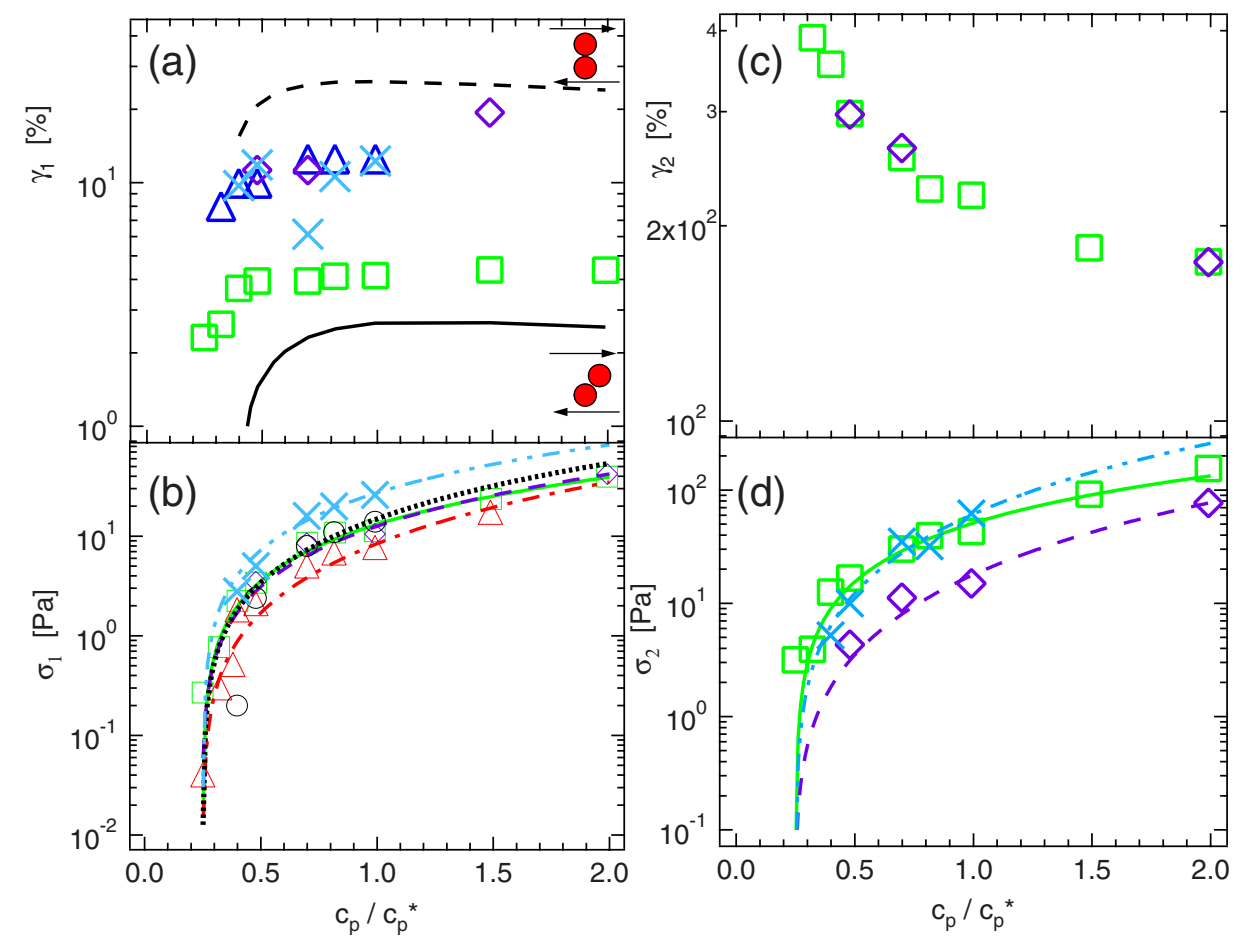

FIG. 18. (a),(c) First and second yield strains $\gamma_{1}$ and $\gamma_{2}$ as a function of polymer concentration $c_{p} / c_{p}^{*}$ extracted from DSSs with frequency $\omega=1 \mathrm{rad} / \mathrm{s}$ as the onset of the sublinear stress-strain relation, $\sigma(\gamma)(\square)$, and the first maximum of the elastic stress, $G^{\prime} \gamma(\gamma)(\triangle)$; the strain at the first and second peak of step rate tests $(\diamond)$; and the maximum recovered strain (x). Lines in (a) represent predictions for the first yield strain $\gamma_{1}$ based on particles which are touching and whose relative initial location leads to the maximum-achievable separation (solid line) or is in the gradient direction (dashed line), as indicated. (b),(d) First and second yield stresses $\sigma_{1}$ and $\sigma_{2}$ as a function of polymer concentration $c_{p} / c_{p}^{*}$ extracted from the onset of the sub-linear stress-strain relation in DSSs $(\square)$, the first and second overshoots or shoulder of the strain-stress dependence in step rate tests $(\diamond)$, the stress at the lowest shear rate in flow experiments $(\triangle)$, the maximum of the creep exponent in step stress (creep) tests $(\bigcirc)$, and the maximum and onset of the plateau value of the maximum recovered strain-stress curve in recovery experiments $(\times)$. Lines represent power-law fits.

model [Fig. 18(a)]. Together with the first yield strain $\gamma_{1}$ that has been extracted from DSSs, we show in Fig. 18(a) the data obtained from the first maximum or shoulder of the elastic stress [Fig. 18(a)], corresponding to the left arrow in Fig. 6(b), the strain at the first and second peaks of step rate tests, and the maximum recovered strain.

The first yield stresses $\sigma_{1}$, which have been determined by different methods, are similar [Fig. 18(b)]. They have already been compared to the power-law dependence $\sigma$ $=\hat{\sigma}\left[\left(c_{p} / c_{p}^{\mathrm{gel}}\right)^{\mu}-1\right]$ which has been derived based on a MCT approach considering the shear-induced reduction of the free-energy barrier [Kobelev and Schweizer (2005a)] (Sec. III D 1). The obtained exponents $1.4 \leq \mu \leq 2.0$ are in good agreement with experimental values for similar systems [Buscall et al. (1987); Gopalakrishnan and Zukoski (2007); Larson (1999); Russel and Grant (2000)] and theoretical predictions [Kobelev and Schweizer (2005a)]. This agreement further supports our interpretation that the first yield point is related to bond breaking.

\section{CONCLUSIONS}

The rheological properties, specifically in the nonlinear regime, of intermediate volume fraction $(\phi=0.4)$ colloid-polymer mixtures have been investigated as a function of 
increasing interparticle attraction. If the attraction is sufficiently strong, colloidal gels are formed which show a complex rheological response with two distinct yielding processes. These two processes are clearly distinguishable in oscillatory and steady shear experiments: in DSSs by two distinct sublinear stress-strain dependencies, in step rate tests by two distinct overshoots or shoulders, and in step stress (creep) tests by an intermediate regime and thus two transitions from creep to flow. We attribute this complex yielding behavior to the heterogeneous structure of the gels, consisting of a network of particle clusters [Dibble et al. (2006); Laurati et al. (2009); Shah et al. (2003c); Smith et al. (2007); Varadan and Solomon (2003)]. Mechanical forces or deformations can thus act on bonds between clusters, thus disrupting the network, and bonds between particles, thus restructuring or fragmenting the clusters. The heterogeneous or cluster structure is thus consistent with the idea of two distinct yielding processes. Two distinct yield points have also been observed in attractive glasses [Pham et al. (2006, 2008)]. In both systems, the first yielding process is linked to bond breaking, while the second yielding process is linked to bond breaking in gels and to elastic cage deformations in attractive glasses.

We propose that the first yielding point corresponds to the shear-induced breaking of intercluster bonds, which disrupts the network. The first yield strain $\gamma_{1}$ was found to be comparable to the range of the attraction, the "bond length." However, it is also affected by the bond strength. We combine both effects in a model which is based on the escape of a particle from the attractive potential of its neighbor, i.e., the bond. Shear drives the particle closer to the edge of the potential and thus decreases the potential barrier and hence the escape time. At the yield strain, the escape time becomes comparable to the residence time at the edge of the potential. The predicted yield strain is in good agreement with the observed first yield strain and its dependence on polymer concentration, which determines the attraction strength and range, and shear frequency, which controls the residence time. This suggests that the model correctly captures the balance between attraction strength and range. Furthermore, the power-law dependence of the first yield stress $\sigma_{1}$ on polymer concentration $c_{p} / c_{p}^{*}$ agrees with a prediction based on the shearinduced reduction of the energy barrier localizing bonded particles [Kobelev and Schweizer (2005a)] and experimental results [Buscall et al. (1987); Gopalakrishnan and Zukoski (2007); Larson (1999)]. The shear-induced breaking of intercluster bonds is also consistent with theoretical and experimental findings in the linear regime [Laurati et al. (2009); Zaccone and Del Gado (2009)].

In the first and second yielding processes, the same kind of bonds is broken on a particle level. However, on a mesoscopic scale they are thought to hold individual particles in a cluster (intracluster bonds) and to connect individual clusters (intercluster bonds), respectively. While the individual bonds are indistinguishable, the number of bonds per particle is expected to be different in the two cases as well as their implications for the structure. Rheology can only provide indirect evidence for this model. Direct support has to be obtained by microscopic structural techniques, such as confocal microscopy or computer simulations. In particular, confocal microscopy studies of the structure of similar colloidal gels [Dibble et al. (2006); Smith (2004)] provide an estimate of the distribution of the number of bonds per particle and its mean as a function of polymer concentration at fixed volume fraction. They indicate that a significant fraction of particles only have a small number of bonds. These particles might represent the connections between neighboring clusters and are expected to be the first to break under shear due to their weaker connectivity. Nevertheless, further microscopic and structural data are required to test the model we propose.

Upon increasing the deformation, we expect intracluster bonds to break, which results in smaller clusters or individual particles. These rearrangements cause a complex rheo- 
logical response at intermediate strains or stresses. The observed second yield strain $\gamma_{2}$ and its dependence on polymer concentration [Fig. 18(c)] reflect the decreasing cluster size with increasing polymer concentration, supporting its connection with yielding of clusters [Smith et al. (2007); Laurati et al. (2009)]. Even above the second yield point, a finite strain is recovered after cessation of shear, indicating that some elastic structure remains. The second yield stress $\sigma_{2}$ also shows a power-law dependence on polymer concentration $c_{p} / c_{p}^{*}$ similar to $\sigma_{1}$ [Fig. 18(d)]. The prefactor of the power-law dependence $\hat{\sigma}$ is however about five times higher than in the case of $\sigma_{1}$, reflecting the difference between intercluster and intracluster bonds.

\section{ACKNOWLEDGMENTS}

This work was funded by the Deutsche Forschungsgemeinschaft (DFG) within the German-Dutch Collaborative Research Centre Sonderforschungsbereich-Transregio 6 (SFB-TR6), Project Section A6, by the Greek General Secretariat for Research through PENED 03E $\Delta 566$, and by the EU Transfer of Knowledge project "Cosines" and the Network of Excellence "SoftComp." We acknowledge N. Koumakis for useful discussions and assistance during the rheological experiments, A. B. Schofield for providing the particles, and M. Fuchs, J. M. Brader, and K. J. Mutch for very stimulating discussions.

\section{References}

Aarts, D. G. A. L., R. Tuinier, and H. N. W. Lekkerkerker, "Phase behaviour of mixtures of colloidal spheres and excluded-volume polymer chains," J. Phys.: Condens. Matter 14, 7551-7561 (2002).

Ballesta, P., R. Besseling, L. Isa, G. Petekidis, and W. C. K. Poon, "Slip and flow of hard-sphere colloidal glasses," Phys. Rev. Lett. 101, 258301 (2008).

Banchio, A. J., and J. F. Brady, “Accelerated Stokesian dynamics: Brownian motion,” J. Chem. Phys. 118, 10323-10332 (2003).

Becu, L., S. Manneville, and A. Colin, "Yielding and flow in adhesive and nonadhesive concentrated emulsions," Phys. Rev. Lett. 96, 138302 (2006).

Bergenholtz, J., W. C. K. Poon, and M. Fuchs, “Gelation in model colloid-polymer mixtures," Langmuir 19, 4493-4503 (2003).

Berry, G. C., "Thermodynamic and conformational properties of polystirene. I. light-scattering studies on dilute solutions of linear polystirenes," J. Chem. Phys. 44, 4550-4564 (1966).

Besseling, R., L. Isa, P. Ballesta, G. Petekidis, M. E. Cates, and W. C. K. Poon, "Shear banding and flowconcentration coupling in colloidal glasses," Phys. Rev. Lett. 105, 268301 (2010).

Brader, J. M., M. E. Cates, and M. Fuchs, "First-principles constitutive equation for suspension rheology," Phys. Rev. Lett. 101, 138301 (2008).

Brader, J. M., T. Voigtmann, M. E. Cates, and M. Fuchs, "Dense colloidal suspensions under time-dependent shear,” Phys. Rev. Lett. 98, 058301 (2007).

Buscall, R., I. J. McGowan, P. D. A. Mills, R. F. Stewart, D. Sutton, L. R. White, and G. E. Yates, "The rheology of strongly-flocculated suspensions,” J. Non-Newtonian Fluid Mech. 24, 183-202 (1987).

Cardinaux, F., T. Gibaud, A. Stradner, and P. Schurtenberger, "Interplay between spinodal decomposition and glass formation in proteins exhibiting short-range attractions," Phys. Rev. Lett. 99, 118301 (2007).

Carrier, V., and G. Petekidis, "Nonlinear rheology of colloidal glasses of soft thermosensitive microgel particles," J. Rheol. 53, 245-273 (2009).

Cates, M. E., "Arrest and flow of colloidal glasses," Ann. Henri Poincare 2, S647-S661 (2003).

Cloitre, M., R. Borrega, and L. Leibler, "Rheological aging and rejuvenation in microgel pastes," Phys. Rev. Lett. 85, 4819-4822 (2000).

Cohen, I., B. Davidovitch, A. B. Schofield, M. O. Brenner, and D. A. Weitz, "Slip, yield and bands in colloidal 
crystals under oscillatory shear,” Phys. Rev. Lett. 97, 215502 (2006).

Conrad, J. C., H. M. Wyss, S. Manley, V. Trappe, K. Miyazaki, L. J. Kaufman, A. Schofield, D. R. Reichman, and D. A. Weitz, "Arrested fluid-fluid phase separation in depletion systems: Implications of the characteristic length on gel formation and rheology," J. Rheol. 54, 421-438 (2010).

Coussot, P., Rheometry of Pastes, Suspensions and Granular Materials (Wiley, New York, 2005).

Crassous, J. J., M. Siebenburger, M. Ballauff, M. Drechsler, D. Hajnal, O. Henrich, and M. Fuchs, "Shear stresses of colloidal dispersions at the glass transition in equilibrium and in flow," J. Chem. Phys. 128, 204902 (2008).

Daniel, C., I. W. Hamley, M. Wilhelm, and W. Mingvanish, "Non-linear rheology of a face-centred cubic phase in a diblock copolymer gel," Rheol. Acta 40, 39-48 (2001).

Dawson, K. A., G. Foffi, M. Fuchs, W. Götze, F. Sciortino, M. Sperl, P. Tartaglia, T. Voigtmann, and E. Zaccarelli, "Mode-coupling theory of colloids with short-range attractions," Phys. Rev. E 63, 011401 (2001).

Derec, C., G. Ducouret, A. Ajdari, and F. Lequex, "Aging and nonlinear rheology in suspensions of polyethylene oxide-protected silica particles," Phys. Rev. E 67, 061403 (2003).

Dibble, C. J., M. Kogan, and M. J. Solomon, "Structure and dynamics of colloidal depletion gels: Coincidence of transitions and heterogeneity," Phys. Rev. E 74, 041403 (2006).

Dyre, J. C., "Master-equation approach to the glass-transition," Phys. Rev. Lett. 58, 792-795 (1987).

Eckert, T., and E. Bartsch, "Re-entrant glass transition in a colloid-polymer mixture with depletion attractions," Phys. Rev. Lett. 89, 125701 (2002).

Ewoldt, R. H., A. E. Hosoi, and G. H. McKinley, "New measures for characterizing nonlinear viscoelasticity in large amplitude oscillatory shear,” J. Rheol. 52, 1427-1458 (2008).

Fleer, G. J., and R. Tuinier, “Analytical phase diagram for colloid-polymer mixtures,” Phys. Rev. E 76, 041802 (2007).

Fuchs, M., and M. Ballauff, "Flow curves of dense colloidal dispersions: Schematic model analysis of the shear-dependent viscosity near the colloidal glass transition,” J. Chem. Phys. 122, 094707 (2005).

Fuchs, M., and M. E. Cates, "Theory of nonlinear rheology and yielding of dense colloidal suspensions," Phys. Rev. Lett. 89, 248304 (2002).

Fuchs, M., and K. S. Schweizer, "Structure of colloid-polymer suspensions," J. Phys.: Condens. Matter 14, R239-R269 (2002).

Furst, E. M., and J. P. Pantina, "Yielding in colloidal gels due to nonlinear microstructure bending mechanics," Phys. Rev. E 75, 050402 (2007).

Gadala-Maria, F., and A. Acrivos, "Shear-induced structure in a concentrated suspension of solid spheres," J. Rheol. 24, 799-814 (1980).

Gopalakrishnan, V., and C. F. Zukoski, "Yielding behavior of thermo-reversible colloidal gels," Langmuir 23, 8187-8193 (2007).

Helgeson, M. E., D. Vlassopoulos, and N. J. Wagner, "Viscoelasticity and shear melting of colloidal star polymer glasses,” J. Rheol. 51, 297-316 (2007).

Hyun, K., E. S. Baik, K. H. Ahn, S. J. Lee, M. Sugimoto, and K. Koyama, "Fourier-transform rheology under medium amplitude oscillatory shear for linear and branched polymer melts," J. Rheol. 51, 1319-1342 (2007).

Hyun, K., S. H. Kim, K. H. Ahn, and S. J. Lee, "Large amplitude oscillatory shear as a way to classify the complex fluids," J. Non-Newtonian Fluid Mech. 107, 51-65 (2002).

Ilett, S. M., A. Orrock, W. C. K. Poon, and P. N. Pusey, "Phase-behavior of a model colloid-polymer mixture," Phys. Rev. E 51, 1344-1352 (1995).

Johnson, W. L., and K. Samwer, "A universal criterion for plastic yielding of metallic glasses with a $(t / t-g)$ $\times(2 / 3)$ temperature dependence," Phys. Rev. Lett. 95, 195501 (2005).

Kobelev, V., and K. S. Schweizer, "Nonlinear elasticity and yielding of depletion gels," J. Chem. Phys. 123, 164902 (2005a).

Kobelev, V., and K. S. Schweizer, "Strain softening, yielding, and shear thinning in glassy colloidal suspensions," Phys. Rev. E 71, 021401 (2005b).

Koumakis, N., and G. Petekidis, "Rheology of frustrated colloidal states: Transition from a colloidal gel to an 
attractive glass," 15th International Congress on Rheology/80th Annual Meeting of the Society-of-Rheology, Monterey, CA (AIP, Melville, NY, 2008), 1027, 1024-1026.

Koumakis, N., and G. Petekidis, "Two step yielding in attractive colloids: transition from gels to attractive glasses," Soft Matter 7, 2456-2470 (2011).

Kramers, H. A., "Brownian motion in a field of force and the diffusion model of chemical reactions," Physica (Amsterdam) 7, 284-304 (1940).

Kroy, K., M. E. Cates, and W. C. K. Poon, "Cluster mode-coupling approach to weak gelation in attractive colloids," Phys. Rev. Lett. 92, 148302 (2004).

Larson, R. G., The Structure and Rheology of Complex Fluids (Oxford University Press, New York, 1999).

Laurati, M., G. Petekidis, N. Koumakis, F. Cardinaux, A. B. Schofield, J. M. Brader, M. Fuchs, and S. U. Egelhaaf, "Structure, dynamics, and rheology of colloid-polymer mixtures: From liquids to gels," J. Chem. Phys. 130, 134907 (2009).

Lee, M. H., and E. M. Furst, "Response of a colloidal gel to a microscopic oscillatory strain,” Phys. Rev. E 77, 041408 (2008).

Lekkerkerker, H. N. W., W. C. K. Poon, P. N. Pusey, A. Stroobants, and P. B. Warren, "Phase-behavior of colloid plus polymer mixtures," Europhys. Lett. 20, 559-564 (1992).

Letwimolnun, W., B. Vergnes, G. Ausias, and P. J. Carreau, "Stress overshoots of organoclay nanocomposites in transient shear flow," J. Non-Newtonian Fluid Mech. 141, 167-179 (2007).

Le Grand, A., and G. Petekidis, "Effects of particle softness on the rheology and yielding of colloidal glasses," Rheol. Acta 47, 579-590 (2008).

Lu, P. J., E. Zaccarelli, F. Ciulla, A. B. Schofield, F. Sciortino, and D. A. Weitz, "Gelation of particles with short-range attraction," Nature (London) 453, 499-503 (2008).

Manley, S., H. Wyss, K. Miyazaki, J. Conrad, V. Trappe, L. J. Kaufmann, D. R. Reichmann, and D. A. Weitz, "Glasslike arrest in spinodal decomposition as a route to colloidal gelation," Phys. Rev. Lett. 94, 238302 (2005).

Mason, T. G., and D. A. Weitz, "Linear viscoelasticity of colloidal hard-sphere suspensions near the glasstransition," Phys. Rev. Lett. 75, 2770-2773 (1995).

McLeish, T., Soft and Fragile Matter, Non-Equilibrium Dynamics, Metastability and Flow (Institute of Physics, Bristol, 2000).

Miyazaki, K., and D. R. Reichman, "Molecular hydrodynamic theory of supercooled liquids and colloidal suspensions under shear,” Phys. Rev. E 66, 050501 (2002).

Miyazaki, K., H. M. Wyss, D. A. Weitz, and D. R. Reichman, "Nonlinear viscoelasticity of metastable complex fluids," Europhys. Lett. 75, 915-921 (2006).

Monthus, C., and J. P. Bouchaud, "Rheological constitutive equation for a model of soft glassy materials," J. Phys. A 29, 3847-3869 (1996).

Osaki, K., T. Inoue, and T. Isomura, "Stress overshoot of polymer solutions at high rates of shear; polystyrene with bimodal molecular weight distribution,” J. Polym. Sci., Part B: Polym. Phys. 38, 2043-2050 (2000).

Pai, V. B., and S. A. Khan, "Gelation and rheology of xanthan/enzyme-modified guar blends," Carbohydr. Polym. 49, 207-216 (2002).

Petekidis, G., A. Moussaid, and P. N. Pusey, "Rearrangements in hard-sphere glasses under oscillatory shear strain,” Phys. Rev. E 66, 051402 (2002a).

Petekidis, G., P. N. Pusey, A. Moussaid, S. U. Egelhaaf, and W. C. K. Poon, "Shear-induced yielding and ordering in concentrated particle suspensions," Physica A 306, 334 (2002b).

Petekidis, G., D. Vlassopoulos, and P. N. Pusey, "Yielding and flow of colloidal glasses," Faraday Discuss. 123, 287-302 (2003).

Petekidis, G., D. Vlassopoulos, and P. N. Pusey, "Yielding and flow of sheared colloidal glasses," J. Phys.: Condens. Matter 16, S3955-S3963 (2004).

Pham, K. N., S. U. Egelhaaf, P. N. Pusey, and W. C. K. Poon, "Glasses in hard spheres with short-range attraction," Phys. Rev. E 69, 011503 (2004).

Pham, K. N., G. Petekidis, D. Vlassopoulos, S. U. Egelhaaf, W. C. K. Poon, and P. N. Pusey, "Yielding behavior of repulsion- and attraction-dominated colloidal glasses," J. Rheol. 52, 649-676 (2008).

Pham, K. N., G. Petekidis, D. Vlassopoulos, S. U. Egelhaaf, P. N. Pusey, and W. C. K. Poon, "Yielding of 
colloidal glasses,” Europhys. Lett. 75, 624-630 (2006).

Pham, K. N., A. M. Puertas, J. Bergenholtz, S. U. Egelhaaf, A. Moussaid, P. N. Pusey, A. B. Schofield, M. E. Cates, M. Fuchs, and W. C. K. Poon, "Multiple glassy states in a simple model system," Science 296, 104-106 (2002).

Poon, W. C. K., "The physics of a model colloid-polymer mixture,” J. Phys.: Condens. Matter 14, R859-R880 (2002).

Poon, W. C. K., K. N. Pham, S. U. Egelhaaf, and P. N. Pusey, “'Unsticking' a colloidal glass, and sticking it again,” J. Phys.: Condens. Matter 15, S269-S275 (2003).

Poon, W. C. K., A. D. Pirie, M. D. Haw, and P. N. Pusey, "Non-equilibrium behaviour of colloid-polymer mixture," Physica A 235, 110-119 (1997).

Pusey, P. N., Liquids, Freezing and Glass Transition (North-Holland, Amsterdam, 1991).

Pusey, P. N., and W. van Megen, "Phase-behavior of concentrated suspensions of nearly hard colloidal spheres," Nature (London) 320, 340-342 (1986).

Pusey, P. N., and W. van Megen, "Observation of a glass-transition in suspensions of spherical colloidal particles,” Phys. Rev. Lett. 59, 2083-2086 (1987).

Rao, R. B., V. L. Kobelev, Q. Li, J. A. Lewis, and K. S. Schweizer, "Nonlinear elasticity and yielding of nanoparticle glasses," Langmuir 22, 2441-2443 (2006).

Ravindranath, S., and S.-Q. Wang, "Large amplitude oscillatory shear behavior of entangled polymer solutions: Particle tracking velocimetric investigation,” J. Rheol. 52, 341-359 (2008).

Renou, F., J. Stellbrink, and G. Petekidis, "Yielding processes in a colloidal glass of soft star-like micelles under large amplitude oscillatory shear (LAOS)," J. Rheol. 54, 1219-1243 (2010).

Rottler, J., and M. O. Robbins, "Shear yielding of amorphous glassy solids: Effect of temperature and strain rate," Phys. Rev. E 68, 011507 (2003).

Rouyer, F., S. Cohen-Addad, M. Vignes-Adler, and R. Hohler, "Dynamics of yielding observed in a threedimensional aqueous dry foam,” Phys. Rev. E 67, 021405 (2003).

Russel, W. B., and M. C. Grant, "Distinguishing between dynamic yielding and wall slip in a weakly flocculated colloidal dispersion," Colloids Surf. 161, 271-282 (2000).

Schaertl, W., and H. Silescu, "Brownian dynamics of polydisperse colloidal hard-spheres-Equilibrium structures and random close packings," J. Stat. Phys. 77, 1007-1025 (1994).

Shah, S. A., Y. L. Chen, and K. S. Schweizer, "Phase behavior and concentration fluctuations in suspensions of hard spheres and nearly ideal polymers," J. Chem. Phys. 118, 3350-3361 (2003a).

Shah, S. A., Y. L. Chen, K. S. Schweizer, and C. F. Zukoski, "Viscoelasticity and rheology of depletion flocculated gels and fluids," J. Chem. Phys. 119, 8747-8760 (2003b).

Shah, S. A., S. Ramakrishnan, Y. L. Chen, K. S. Schweizer, and C. F. Zukoski, "Microstructure of dense colloid-polymer suspensions and gels," J. Phys.: Condens. Matter 15, 4751-4778 (2003c).

Siebenburger, M., M. Fuchs, H. Winter, and M. Ballauff, "Viscoelasticity and shear flow of concentrated, noncrystallizing colloidal suspensions: Comparison with mode-coupling theory," J. Rheol. 53, 707-726 (2009).

Smith, P. A., "Colloidal gels under oscillatory shear," Ph.D. thesis, University of Edinburgh, 2004.

Smith, P. A., G. Petekidis, S. U. Egelhaaf, and W. C. K. Poon, "Yielding and crystallization of colloidal gels under oscillatory shear," Phys. Rev. E 76, 041402 (2007).

Sollich, P., "Rheological constitutive equation for a model of soft glassy materials," Phys. Rev. E 58, 738-759 (1998).

Stokes, J. R., and W. J. Frith, "Rheology of gelling and yielding soft matter systems," Soft Matter 4, 1133-1140 (2008).

Tapadia, P., S. Ravindranath, and S.-Q. Wang, "Banding in entangled polymer fluids under oscillatory shearing," Phys. Rev. Lett. 96, 196001 (2006).

van Aken, B., P. de Hey, and J. Sietsma, "Structural relaxation and plastic flow in amorphous La50A125Ni25," Mater. Sci. Eng., A 278, 247-254 (2000).

Varadan, P., and M. J. Solomon, "Direct visualization of long-range heterogeneous structure in dense colloidal gels," Langmuir 19, 509-512 (2003).

Verduin, H., and J. K. G. Dhont, "Phase-diagram of a model adhesive hard-sphere dispersion," J. Colloid 
Interface Sci. 172, 425-437 (1995).

Verhaegh, N. A. M., D. Asnaghi, H. N. W. Lekkerkerker, M. Giglio, and L. Cipelletti, "Transient gelation by spinodal decomposition in colloid-polymer mixtures," Physica A 242, 104-118 (1997).

Walls, H. J., S. B. Caines, A. M. Sanchez, and S. A. Kahn, "Yield stress and wall slip phenomena in colloidal silica gels," J. Rheol. 47, 847 (2003).

Woutersen, A. T. J. M., and C. G. de Kruif, “The rheology of adhesive hard-sphere dispersions,” J. Chem. Phys. 94, 5739-5750 (1991).

Wyss, H. M., K. Miyazaki, J. Mattsson, Z. Hu, D. R. Reichman, and D. A. Weitz, "Strain-rate frequency superposition: A rheological probe of structural relaxation in soft materials," Phys. Rev. Lett. 98, 238303 (2007).

Yang, M. C., L. E. Scriven, and C. W. Macosko, "Some rheological measurements on magnetic iron-oxide suspensions in silicone oil,” J. Rheol. 30, 1015-1029 (1986).

Zaccone, A., and E. Del Gado, "Elasticity of arrested short-ranged attractive colloids: Homogeneous and heterogeneous glasses," Phys. Rev. Lett. 103, 208301 (2009).

Zausch, J., and J. Horbach, "The build-up and relaxation of stresses in a glass-forming soft-sphere mixture under shear: A computer simulation study," EPL 88, 60001 (2009).

Zausch, J., J. Horbach, M. Laurati, S. U. Egelhaaf, J. M. Brader, T. Voigtmann, and M. Fuchs, "From equilibrium to steady state: the transient dynamics of colloidal liquids under shear," J. Phys.: Condens. Matter 20, 404210 (2008). 